\title{
Les diodes laser : des principes aux développements récents
}

\author{
B. de Cremoux
}

Laboratoire Central de Recherches Thomson-CSF, Domaine de Corbeville, 91404 Orsay cedex, France

\section{INTRODUCTION}

La diode Laser, encore appelée Laser à semi-conducteur, Laser à injection, Laser à jonction, Laser à hétérojonctions, Laser à puits quantiques ou même Laser à diode (probablement traduction - trop - directe de l'anglais "Laser diode") est une diode à semi-conducteur, plus précisément une diode électroluminescente, conçue et utilisée de façon à exploiter l'effet LASER.

Démontrée pour la première fois en 1962 [1,2], elle est en 1994 un composant optoélectronique produit industriellement par quantités annuelles de plusieurs millions pour certains types comme le montre le tableau I. L'activité économique correspondante est très importante puisque le marché mondial des diodes Laser en 1993 a été de 231 M\$ soit $26 \%$ du marché de tous types de Lasers. Une croissance de 14\% est prévue pour 1994 [3]

\begin{tabular}{|c|c|c|c|}
\hline $\begin{array}{c}\text { Applications } \Rightarrow \\
\text { Types } \Omega\end{array}$ & Télécom. & Enreg./Lecture & Imprimantes \\
\hline$\lambda<700 \mathrm{~nm}$ & & $\begin{array}{l}10000 \\
1000 \mathrm{k \$}\end{array}$ & $\begin{array}{l}2100 \\
200 \mathrm{k} \$\end{array}$ \\
\hline $\begin{array}{l}750<\lambda<950 \mathrm{~nm} \\
\mathrm{P}<10 \mathrm{~mW}\end{array}$ & & $\begin{array}{l}67000000 \\
74000 \mathrm{k \$} \\
\end{array}$ & \\
\hline $\begin{array}{l}750<\lambda<950 \mathrm{~nm} \\
\mathrm{P}>10 \mathrm{~mW}\end{array}$ & $\begin{array}{l}300 \\
450 \mathrm{kS} \\
\end{array}$ & & $\begin{array}{l}2300000 \\
7500 \mathrm{k \$} \\
\end{array}$ \\
\hline $950<\lambda<1550 \mathrm{~nm}$ & $\begin{array}{l}6200000 \\
90000 \mathrm{k \$}\end{array}$ & & \\
\hline
\end{tabular}

Tableau I : Quantités et chiffres d'affaires des principaux segments du marché mondial des diodes Laser [3]. 
On se propose dans la suite de donner au lecteur une vue générale de l'état de l'art dans le domaine, sans développements physico-mathématiques compliqués, mais en fournissant néanmoins les outils permettant de repérer les ordres de grandeur des paramètres régissant la conception et caractérisant le fonctionnement de ces dispositifs ainsi que de suivre leur évolution future. Dans ce but, on rappellera d'abord les principes généraux relevant de la physique des semi-conducteurs (émission de rayonnement dans les semi-conducteurs, matériaux disponibles, excitation par injection dans les structures de diodes à jonction). On décrira ensuite quelques structures de diodes Laser de référence, les premières à avoir été développées dans les années 70 comme émetteurs pour les télécommunications sur fibre optique et les principales étapes de leur fabrication. On présentera alors leurs caractéristiques fonctionnelles en essayant de dégager quantitativement leur relation aux paramètres définissant les structures. On exposera enfin les voies majeures de diversification à partir des composants de référence, déterminant l'extension rapide du domaine des performances accessibles.

\section{2. ÉMISSION DE RAYONNEMENT PAR LES SEMI-CONDUCTEURS}

2.1 Densité d'états électroniques dans les semi-conducteurs : bandes d'énergie, niveaux d'impureté, trous $[4,5]$

Dans un solide cristallin parfait, assemblage régulier d'atomes aux noeuds d'un réseau tridimensionnel périodique, les états d'énergie des électrons sont répartis en bandes permises et bandes interdites. Pour les isolants et les semi-conducteurs, à la température de $0 \mathrm{~K}$, tous les états de la dernière bande contenant des électrons, appelée bande de valence (BV), sont occupés, tandis que les états de la bande immédiatement supérieure, appelée bande de conduction (BC), sont vides (Fig. 1).

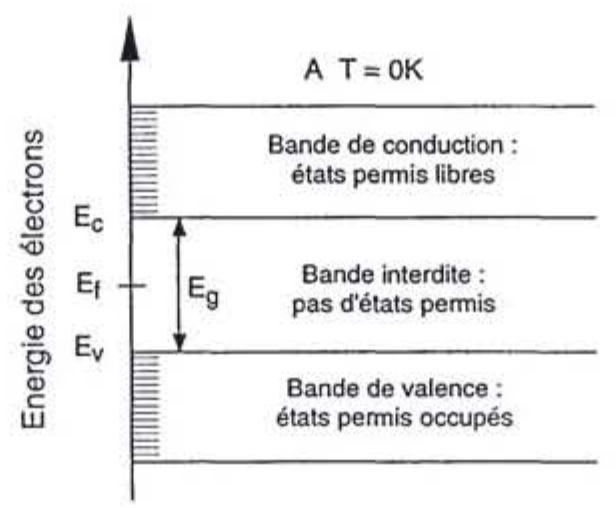

Figure 1: Bandes d'énergies des semi-conducteurs à $0 \mathrm{~K}$

L'écart énergétique entre bande de valence et bande de conduction est appelé largeur de bande interdite (energy band gap ou simplement gap en anglais) et noté $\mathrm{E}_{\mathrm{g}}$. Il est mesuré le plus souvent en électronvolt $\left(1 \mathrm{eV}=1,610^{-19}\right.$ joule). C'est un paramètre fondamental déterminant les propriétés électroniques et optiques des semi-conducteurs. En particulier, il permet de distinguer quantitativement les isolants des semi-conducteurs, ces derniers ayant un 
gap inférieur à quelques $\mathrm{eV}$. On peut rappeler à ce point que la relation entre l'énergie hv des photons exprimée en $\mathrm{eV}$ et leur longueur d'onde $\lambda$ exprimée en micron est donnée par :

$$
\mathrm{h} v \mathrm{x} \lambda=1,24(\mathrm{eV} \times \mu \mathrm{m})
$$

Les écarts à la perfection du réseau cristallin ont pour effet d'introduire des niveaux électroniques permis dans la bande interdite. En particulier certains atomes d'impuretés, différents de ceux constituant la matrice cristalline, peuvent être substitués volontairement à ceux-ci par dopage :

- Les donneurs, possédant un électron de plus sur leur couche périphérique, créent des niveaux voisins de la BC et peuvent lui céder cet électron permettant de modifier la conductivité du matériau sur plusieurs ordres de grandeur. On obtient ainsi un semiconducteur de type $N$.

- Les accepteurs, possédant un électron de moins, créent des niveaux voisins de la BV et peuvent en recevoir un électron, libérant un état électronique. Ces états libres se comportent comme des charges positives, appelées trous, qui peuvent transporter du courant électrique. On obtient alors un semi-conducteur de type $P$.

\subsection{Occupation des états électroniques : statistique de FERMI [4,5]}

Les électrons sont régis par la statistique de FERMI et la probabilité d'occupation des états d'énergie $\mathrm{E}$ est donnée à l'équilibre thermodynamique à une température $\mathrm{T}$ par la formule :

$$
f(E)=1 /\left(1+\exp \left(\left(E-E_{f}\right) / k T\right)\right)
$$

oủ $\mathrm{E}_{\mathrm{f}}$ est appelé niveau de FERMl et $\mathrm{k}$ est la constante de BOLTZMAN $\left(\mathrm{k}=1,3810^{-23} \mathrm{~J} / \mathrm{K}\right)$. A la température ambiante de $300 \mathrm{~K}$ l'énergie $\mathrm{kT}$ vaut $26 \mathrm{meV}$, significativement plus petite que le gap des semi-conducteurs usuels $(\sim 1 \mathrm{eV})$.

Les allures de la fonction de FERMI à $0 \mathrm{~K}$ et à température non nulle sont représentées sur la figure 2 .

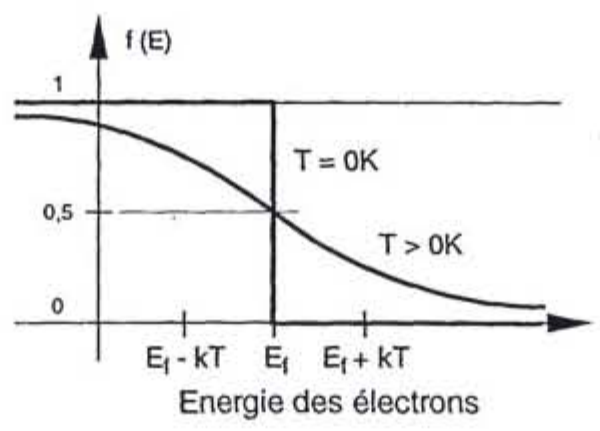

Figure 2: Fonction de Fermi à $\mathrm{T}=0 \mathrm{~K}$ et à $\mathrm{T}>0 \mathrm{~K}$.

- A T $=0 \mathrm{~K}$, tous les états d'énergie inférieure à $\mathrm{E}_{\mathrm{f}}$ (bande de valence) sont occupés, tous les états d'énergie supérieure à $\mathrm{E}_{\mathrm{f}}$ (bande de conduction) sont libres. Il en résulte que le semi-conducteur est en fait isolant 
- A T $>0 \mathrm{~K}$ quelques électrons ont libéré des états de la BV et occupent des états de la BC. Un semi-conducteur non-dopé ou intrinsèque peut ainsi conduire le courant, sa conductivité électrique due à la fois aux électrons et aux trous varie exponentiellement avec $-\mathrm{E}_{\mathrm{g}} / 2 \mathrm{kT}$.

Un matériau semi-conducteur peut être amené hors équilibre thermodynamique par différents moyens, et le plus souvent les populations d'électrons et de trous peuvent être considérées chacune comme en équilibre dans sa bande respective. En effet le temps de mise en équilibre des électrons entre eux et des trous entre eux (temps de relaxation intrabande) est de l'ordre de $10^{-12} \mathrm{~s}$, alors que le temps de mise en équilibre des électrons avec les trous (temps de relaxation interbande ou durée de vie) est généralement supérieur à $10^{-9} \mathrm{~s}$. Les fonctions de FERMI décrivant les occupations de la $\mathrm{BC}$ et de la BV comprennent alors des pseudo-niveaux de FERMI $\mathrm{F}_{\mathrm{n}}$ et $\mathrm{F}_{\mathrm{p}}$ distincts pour les électrons et pour les trous au lieu du niveau unique $\mathrm{E}_{\mathrm{f}}$.

\subsection{Transitions entre états électroniques [6,7]}

\subsubsection{Transitions radiatives, émission et absorption de lumière par un semi-conducteur}

Les interactions entre rayonnement (photons) et matière (électrons) mis en jeu dans les diodes Laser sont représentées schématiquement sur la figure 3 . On y distingue

- l'absorption, où un photon incident cède son énergie à un électron de la BV et le fait "monter" dans la BC, créant une paire électron-trou, c'est un processus de génération radiative;

- l'émission spontanée où un électron "redescend" spontanément sur un état libre de la BV, annihilant une paire électron-trou, c'est un processus de recombinaison radiative;

- l'émission stimulée où un photon incident induit la recombinaison radiative d'une paire électron-trou. Le photon émis a même phase et même direction que le photon incident. C'est le mécanisme découvert par EINSTEIN en 1917 par lequel le rayonnement peut être amplifié et qui est exploité dans tout type de LASER.

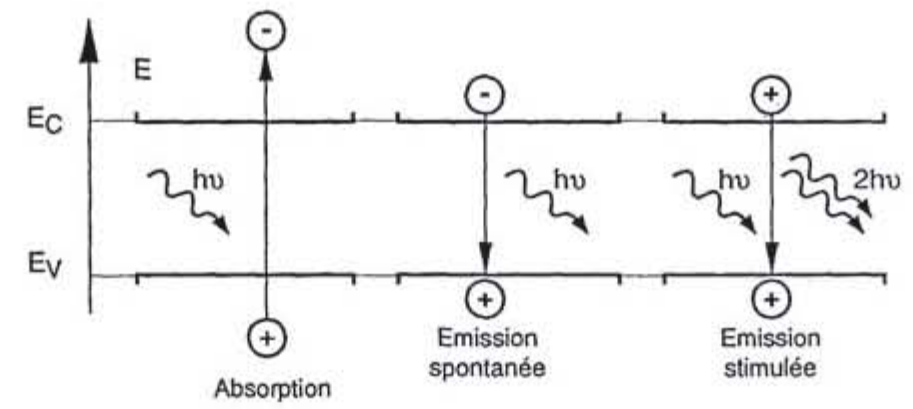

Figure 3 : Absorption et émission de lumière dans un semi-conducteur.

Deux conséquences résultent de ces processus :

- Seuls sont absorbés les photons d'énergie supérieure au gap : $h v \geq \mathrm{E}_{\mathrm{g}}$.

- Les photons émis ont une énergie légèrement supérieure $(\sim \mathrm{kT})$ à celle du gap.

L'émission spontanée peut être caractérisée par une vitesse d'émission spontanée qui mesure 
le nombre de photons émis par unité de temps et par unité de volume du semi-conducteur. Elle est liée aux densités d'électrons et de trous par la relation suivante :

$$
\mathrm{R}_{\mathrm{sp}}=\mathrm{B} \mathrm{n} \mathrm{p}
$$

où $\mathrm{n}$ et $\mathrm{p}$ sont respectivement les densités d'électrons et de trous par unité de volume et $\mathrm{B}$ est appelé coefficient de recombinaison radiative. Son ordre de grandeur pour les semiconducteurs utilisés dans les diodes Laser est : $\mathrm{B}=10^{-10} \mathrm{~cm}^{-3}$ à $300 \mathrm{~K}$.

Un paramètre plus simple utilisé pour caractériser dans une approximation linéaire la recombinaison spontanée est la durée de vie radiative $\tau_{\mathrm{T}}$ qui lie $\mathrm{R}_{\mathrm{Sp}}$ à la densité de porteurs minoritaires (électrons dans un matériau de type $\mathrm{P}$ et vice versa) par la formule

$$
\mathrm{R}_{\mathrm{sp}}=\mathrm{n} / \tau_{\mathrm{r}}=-\mathrm{dn} / \mathrm{dt}
$$

On a alors :

$$
\tau_{\mathrm{r}}=1 / \mathrm{B} \mathrm{p}_{\mathrm{o}}
$$

pour un matériau $\mathrm{P}$ oủ la densité de trous à l'équilibre est $\mathrm{p}_{\mathrm{O}}$. Ainsi pour du matériau dopé à $10^{18} \mathrm{~cm}^{-3}$, la durée de vie radiative des électrons est de $10 \mathrm{~ns}$.

L'absorption et le gain d'émission stimulée peuvent être mesurés en $\mathrm{cm}^{-1}$ par le même coefficient $\mathrm{g}$ défini par

$$
\mathrm{g}=\mathrm{dF} / \mathrm{F} \mathrm{dx}
$$

où $\mathrm{F}$ est le flux de photons se dirigeant suivant la direction $\mathrm{x}$.

$\mathrm{g}$ est négatif pour un matériau absorbant à l'équilibre et positif si le niveau d'excitation est suffisant. Ces situations sont montrées sur la figure 4 où sont tracées schématiquement les courbes donnant le gain en fonction de l'énergie hv des photons pour différentes valeurs de la densité d'électrons en excès par rapport à l'équilibre et de l'écart entre les pseudo-niveaux de FERMI.

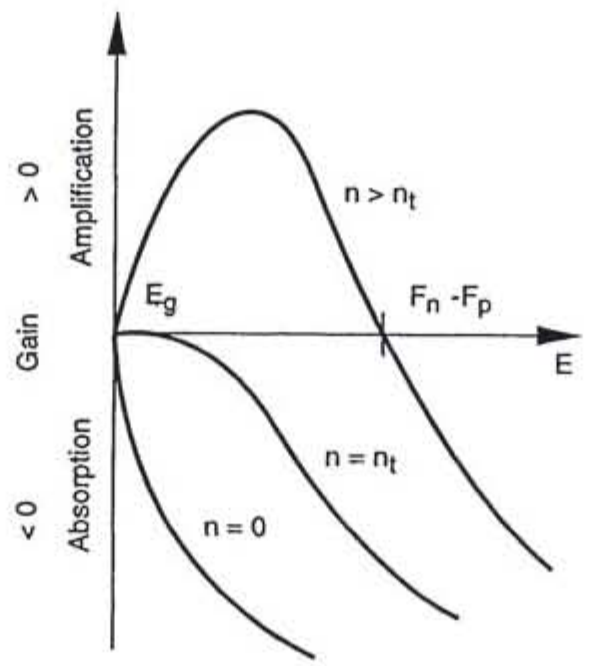

Figure 4 : Gain d'émission stimulée pour différents niveaux d'excitation. 
Pour $\mathrm{n}=0$, le matériau est toujours absorbant. Pour $\mathrm{n}=\mathrm{n}_{\mathrm{t}}$, densité d'électrons à la transparence (ordre de grandeur : $\mathrm{n}_{\mathrm{t}}=10^{18} \mathrm{~cm}^{-3}$ ), le matériau est juste transparent pour hv $=\mathrm{E}_{\mathrm{g}}$. Enfin pour $\mathrm{n}>\mathrm{n}_{\mathrm{t}}$ le matériau amplifie les photons d'énergies hv telles que

$$
\mathrm{E}_{\mathrm{g}}<\mathrm{hv}<\mathrm{F}_{\mathrm{n}}-\mathrm{F}_{\mathrm{p}} \quad \text { (Condition de BERNARD et DURAFFOURG) }
$$

\subsubsection{Transitions non-radiatives, processus SRH, recombinaison AUGER.}

Il existe aussi des transitions non-radiatives dans lesquelles les énergies, absorbée à la génération d'une paire électron-trou ou dissipée à sa recombinaison, sont échangées finalement sous forme de phonons, quanta de vibration du réseau cristallin, en d'autres termes de chaleur.

Le processus de recombinaison SHOCKLEY-READ-HALL (SRH) est schématisé sur la figure 5a. Il fait appel à des niveaux d'énergie voisins du milieu du gap (niveaux dits profonds, dus à certains défauts cristallins ou à certaines impuretés) qui peuvent échanger des électrons à la fois avec la BC et avec la BV. L'efficacité de ce processus dépend de la densité des niveaux profonds et par suite, de la qualité du matériau utilisé.

Le processus de recombinaison AUGER est représenté sur la figure $5 b$ dans le cas particulier du processus électron-électron-trou (e-e-t). L'énergie de la recombinaison est cédée à un deuxième électron qui "monte" dans la $\mathrm{BC}$ puis cède cette énergie au réseau cristallin. Un tel événement nécessite la présence de trois particules et la vitesse de recombinaison AUGER, $R_{a}$ est donnée par la relation suivante :

$$
\mathrm{R}_{\mathrm{a}}=\mathrm{C} \mathrm{n}^{2} \mathrm{p}
$$

où $\mathrm{C}$ est le coefficient de recombinaison AUGER.

La valeur de $\mathrm{C}$, et donc l'efficacité de ce processus, est une caractéristique intrinsèque du matériau et non de sa pureté.

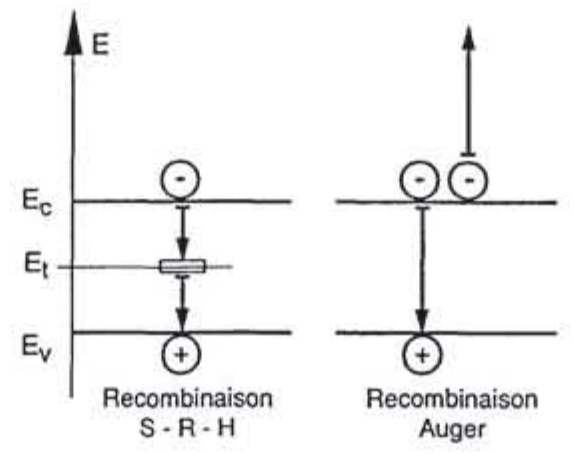

Figure 5 : Recombinaison non-radiative par les processus Schockley-Read-Hall (a) et Auger (5b).

L'ensemble des mécanismes de génération et de recombinaison non-radiatifs peut être caractérisé dans une approximation linéaire par une durée de vie non-radiative $\tau_{\mathrm{n} r}$, la vitesse de recombinaison non-radiative s'écrivant alors :

$$
\mathrm{R}_{\mathrm{nr}}=\mathrm{n} / \tau_{\mathrm{nr}}
$$




\subsubsection{Rendement de recombinaison radiative, durée de vie totale}

Les processus radiatifs et non-radiatifs intervenant simultanément, on utilise deux paramètres décrivant le fonctionnement des émetteurs électroluminescents, diodes électroluminescentes comme diodes Laser et prenant en compte les deux types de mécanismes. En effet la vitesse de recombinaison totale est :

$$
\mathrm{R}=\mathrm{R}_{\mathrm{sp}}+\mathrm{R}_{\mathrm{nr}}
$$

- Le rendement interne de recombinaison radiatif $\eta_{\mathrm{i}}$ est défini par :

$$
\eta_{\mathrm{i}}=\mathrm{R}_{\mathrm{sp}} / \mathrm{R}=\tau_{\mathrm{nr}} /\left(\tau_{\mathrm{r}}+\tau_{\mathrm{nr}}\right)
$$

- La durée de vie totale $\tau$ est définie par :

$$
\tau=\mathrm{n} / \mathrm{R}=\tau_{\mathrm{r}} \tau_{\mathrm{nr}} /\left(\tau_{\mathrm{r}}+\tau_{\mathrm{nr}}\right)
$$

Pour les "bons" matériaux où la recombinaison est essentiellement radiative, $\eta_{\mathrm{i}}$ est voisin de $100 \%$ et $\tau$ approche $\tau_{\mathrm{r}}$.

\section{MATÉRIAUX SEMI-CONDUCTEURS}

\subsection{Matériaux offerts par la nature}

L'extrait suivant du tableau de MENDELEIEFF montre les principaux éléments entrant dans la composition des matériaux semi-conducteurs.

$\begin{array}{ccccc}\text { II } & \text { III } & \text { IV } & \text { V } & \text { VI } \\ & \mathrm{B} & \mathrm{C} & \mathrm{N} & \\ \mathrm{Mg} & \mathrm{Al} & \mathrm{Si} & \mathrm{P} & \mathrm{S} \\ \mathrm{Zn} & \mathrm{Ga} & \mathrm{Ge} & \mathrm{As} & \mathrm{Se} \\ \mathrm{Cd} & \mathrm{In} & \mathrm{Sn} & \mathrm{Sb} & \mathrm{Te} \\ \mathrm{Hg} & & \mathrm{Pb} & & \end{array}$

On y reconnaît les éléments de la colonne IV, dont le Germanium, ancêtre des semiconducteurs et le Silicium, matériau de base de tous les circuits intégrés.

Les composés III-V sont constitués d'éléments des colonnes III et V, leur prototype est le composé binaire Arséniure de Gallium, GaAs, de structure cristalline très voisine de celle du Germanium et dont le gap est de 1,4 eV. Avec les 3 éléments III Al, Ga et In et les 3 éléments $\mathrm{V} P$, As et $\mathrm{Sb}$ il est possible de faire 9 composés binaires de ce type qui ont des gaps allant de 0,2 à $2,4 \mathrm{eV}$.

Les III-V permettent également de faire des composés ternaires, dont le prototype est $\mathrm{Ga}_{1-\mathrm{x}} \mathrm{Al}_{\mathrm{x}} \mathrm{As}$, qui peut être considéré comme une solution solide de $\mathrm{GaAs}$ et $\mathrm{AlAs}$. Le 
paramètre de composition $\mathrm{x}$ permet de faire varier de façon continue les propriétés du matériau, en particulier son gap et son paramètre cristallin qui caractérise la dimension de la maille élémentaire du réseau cristallin.

Il est également possible d'élaborer des composés quaternaires comme $\mathrm{Ga}_{\mathrm{x}} \mathrm{In}_{1-\mathrm{x}} \mathrm{As}_{\mathrm{y}} \mathrm{P}_{1-\mathrm{y}}$ ou $\mathrm{Al}_{1-\mathrm{x}-\mathrm{y}} \mathrm{Ga}_{\mathrm{x}} \mathrm{In}_{\mathrm{y}} \mathrm{P}$ où les 2 paramètres de composition $\mathrm{x}$ et $\mathrm{y}$ permettent d'ajuster indépendamment de façon continue le gap et le paramètre cristallin du matériau.

A côté des III-V, les composés II-VI comme $\mathrm{ZnSeTe}$, et IV-VI comme PbSnTe permettent également de faire des diodes Laser. Mais seuls les composés III-V, et dans une moindre mesure IV-VI, ont une métallurgie suffisamment avancée pour avoir débouché sur des composants commercialisés.

\subsection{Notions d'épitaxie $[6,7]$}

La partie utile des diodes Laser est toujours constituée par un empilement de couches minces (moins de $3 \mu \mathrm{m}$ ) de matériaux semi-conducteurs différant par leur composition et/ou leur type de conductivité. Ces couches sont déposées par les techniques d'épitaxie sur un support "épais" (quelques centaines de microns), généralement en composé binaire, qui a deux fonctions :

- support mécanique des couches utiles,

- modèle cristallin pour les couches épitaxiales au moment de leur élaboration.

L'obtention de couches sans défauts nécessite que, sauf pour les épaisseurs très faibles $(<10 \mathrm{~nm})$, l'écart relatif entre la maille cristalline du substrat et celle d'une couche épitaxiale soit très faible, typiquement :

$$
|\Delta \mathrm{a} / \mathrm{a}|<10^{-3}
$$

où $\Delta \mathrm{a}$ est la différence entre les paramètres cristallins de la couche et du substrat et a est le paramètre cristallin du substrat.

Il est cependant possible d'obtenir des couches désadaptées sans défauts et par suite contraintes (matériaux pseudomorphiques), en dessous d'une épaisseur critique dépendant du degré de désadaptation, ce qui augmente encore l'éventail des matériaux utilisables et est mis à profit dans les diodes Laser à puits quantiques considérées plus loin.

\subsection{Famille GaAlAs [6]}

Les composés ternaires de la famille GaAlAs ont une maille cristalline satisfaisant à la condition précédente pour toutes les valeurs du paramètre de composition $\mathrm{x}$. Ils sont donc préparés par épitaxie sur des substrats en GaAs, que l'on sait obtenir avec de grandes surfaces (diamètre supérieur à 2 pouces).

La figure 6 montre les variations du gap de GaAlAs avec le paramètre de composition $\mathrm{x}$. Ce diagramme a un point anguleux pour $\mathrm{x}=0,4$ correspondant à un changement de nature de la structure de bande :

- pour $\mathrm{x}<0,4$ le matériau est dit à bande interdite directe et la probabilité de transition radiative est élevée;

- pour $\mathrm{x}>0,4$ le matériau est à bande interdite indirecte et ne permet pas de faire des émetteurs électroluminescents efficaces.

Avec ces limitations, ces matériaux peuvent donc être obtenus avec des gaps directs compris entre 1,42 et $1,9 \mathrm{eV}$ susceptibles d'émettre des photons de longueurs d'ondes comprises respectivement entre 870 et $650 \mathrm{~nm}$. 


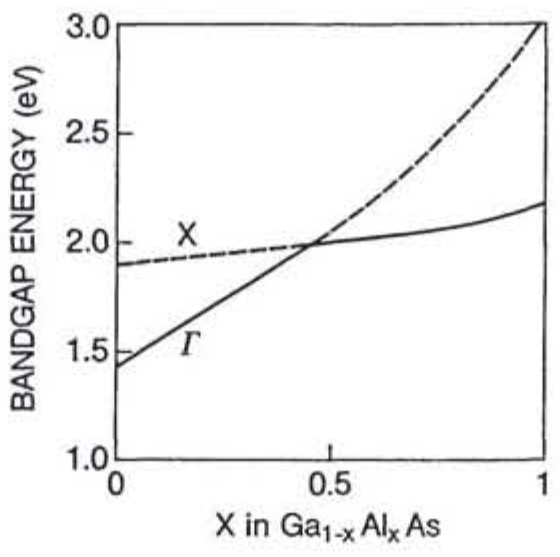

Figure 6: Variation du gap de GaAlAs avec le paramètre de composition x.

\subsection{Famille GaInAsP [7]}

Les propriétés des composés quaternaires sont représentées dans le diagramme de la figure 7 où sont portés, en abscisse le paramètre de composition $\mathrm{x}$ et en ordonnée le paramètre $\mathrm{y}$, de sorte qu'un point du carré représente une composition de la famille. Il est alors possible de tracer dans ce diagramme les courbes iso-paramètre cristallin et les courbes iso-gap.

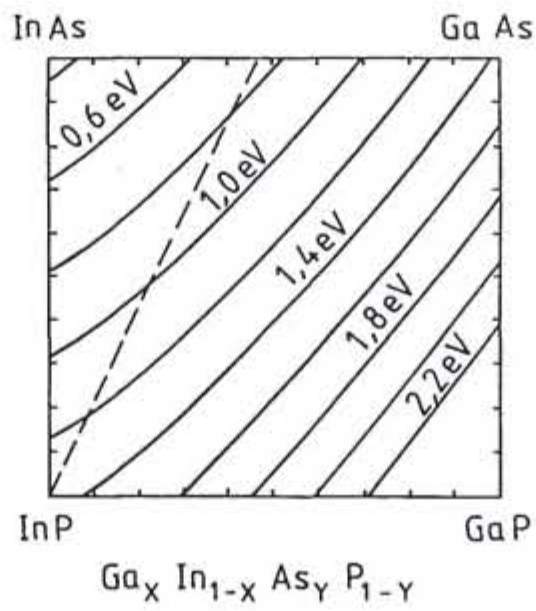

Figure 7 : Courbes iso-gap et iso-paramètre de maille cristalline de GaInAsP 
Il ressort de la figure que, en conservant l'adaptation à des substrats en Phosphure d'Indium, InP, il est possible d'élaborer par épitaxie des matériaux à gaps directs compris entre 1,35 et $0,75 \mathrm{eV}$ qui correspondent à des longueurs d'ondes de photons comprises respectivement entre 930 et $1650 \mathrm{~nm}$.

Dans la même famille, il est possible d'utiliser des substrats en GaAs et de couvrir les gaps de 1,42 à $2,0 \mathrm{eV}$ et les longueurs d'ondes correspondantes.

\section{EXCITATION PAR INJECTION, DIODES À JONCTION, HÉTÉROJONCTIONS}

\subsection{Jonction P-N à l'équilibre et en polarisation directe $[4,5]$}

Une homojonction $\mathrm{P}-\mathrm{N}$ est la réunion de deux semi-conducteurs de même composition et de types de dopage différents, par exemple GaAs de type $\mathrm{P}$ et GaAs de type N. Dans le premier, le niveau de FERMI est proche de la BV, dans le second, il est proche de la BC. Or la thermodynamique exige qu'à l'équilibre, la jonction étant non polarisée, le niveau de Fermi soit constant dans toute la structure. Il en résulte que le profil des bords de bandes de valence et de conduction a l'allure représentée sur la figure $8 \mathrm{a}$; il existe au voisinage de la jonction une zone désertée de porteurs libres, les trous du matériau $\mathrm{P}$ et les électrons du matériau $\mathrm{N}$ sont séparés par les barrières de potentiel représentées et ne peuvent se recombiner.

La polarisation directe d'une jonction consiste à appliquer une tension positive $\mathrm{V}$ au matériau $P$, ce qui a pour effet de diminuer ces barrières de $q V\left(q=1,610^{-19}\right.$ coulomb), sur un diagramme tel que celui de la figure $8 \mathrm{~b}$ où le sens positif correspond aux énergies des électrons. Les trous et les électrons peuvent alors diffuser (transport sous l'effet d'un gradient de concentration) respectivement vers les régions $\mathrm{N}$ et $\mathrm{P}$ et se recombiner au voisinage de la jonction où il y a à la fois des trous et des électrons en excès par rapport à l'équilibre. Pour chaque paire électron-trou recombinée, il passe en régime permanent une charge élémentaire dans le circuit oủ est insérée la diode. Dans la région de recombinaison, le niveau de FERMI, unique à l'équilibre, se dédouble en deux pseudo-niveaux de FERMI dont l'écart, à la jonction, est égal à qV soit :

$$
\mathrm{q} V=\mathrm{F}_{\mathrm{n}}-\mathrm{F}_{\mathrm{p}}
$$

La caractéristique I/V (courant/tension) de la jonction est donnée par la célèbre formule de SHOCKLEY :

$$
I=I_{S}(\exp (q V / k T)-1)
$$

oủ $\mathrm{I}_{\mathrm{S}}$ est appelé courant de saturation.

\subsection{Double hétérojonction [6,7]}

Dans le cas d'une homojonction l'épaisseur de la zone de recombinaison, ou zone active, est déterminée par les mécanismes de diffusion et de recombinaison et a pour ordre de grandeur la somme des longueurs de diffusion des électrons et des trous, soit quelques microns. C'est une caractéristique des matériaux qu'il n'est pas possible de diminuer significativement.

Un moyen d'ajuster par construction l'épaisseur de zone active est de réaliser par épitaxie une structure à double hétérojonction $(\mathrm{DH})$ telle que décrite sous polarisation directe à la figure $8 \mathrm{c}$. Elle comprend une couche du matériau devant constituer la zone active, par exemple $\mathrm{GaAs}$, entre deux couches de matériaux de types respectivement $\mathrm{P}$ et $\mathrm{N}$, par exemple $\mathrm{Ga}_{0,7} \mathrm{Al}_{0,3}$ As. Les jonctions $\mathrm{GaAs}-\mathrm{GaAlAs}$, appelées hétérojonctions parce qu'entre des 
matériaux de gaps différents, constituent des barrières de potentiel pour les électrons et les trous, c'est le confinement électronique. Les moyens modernes d'épitaxie permettent ainsi de réaliser des couches actives dont l'épaisseur peut être aussi faible que quelques couches atomiques et donc inférieure à un nanomètre.

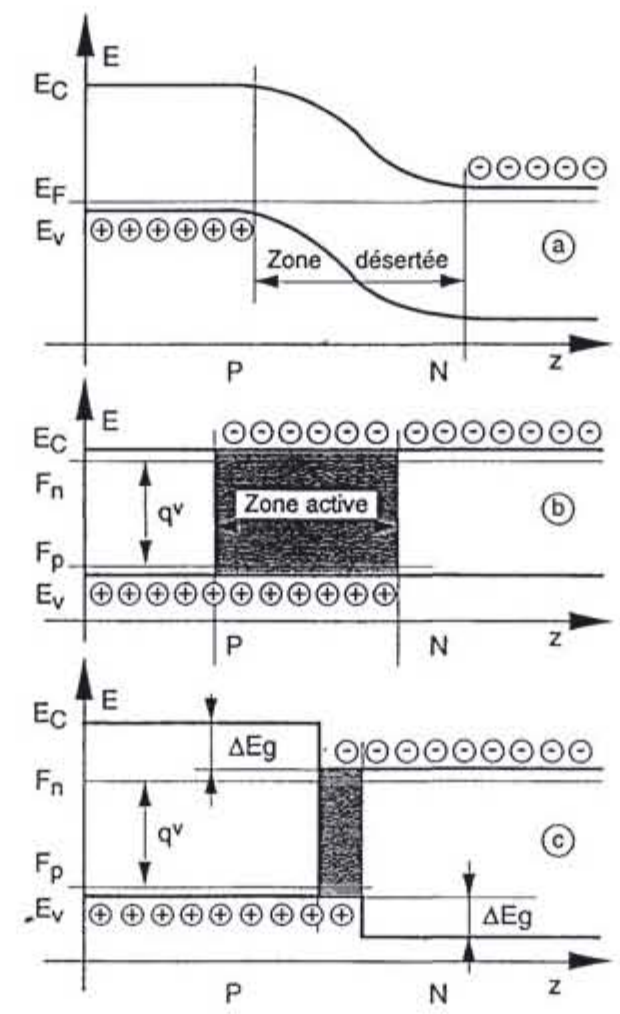

Figure 8 : Profils de bande : homo-jonction à $\mathrm{V}=0$ (8a), homo-jonction à $\mathrm{V}>0(8 \mathrm{~b})$, et doublehétérojonction à $\mathrm{V}>0(\mathrm{8c})$.

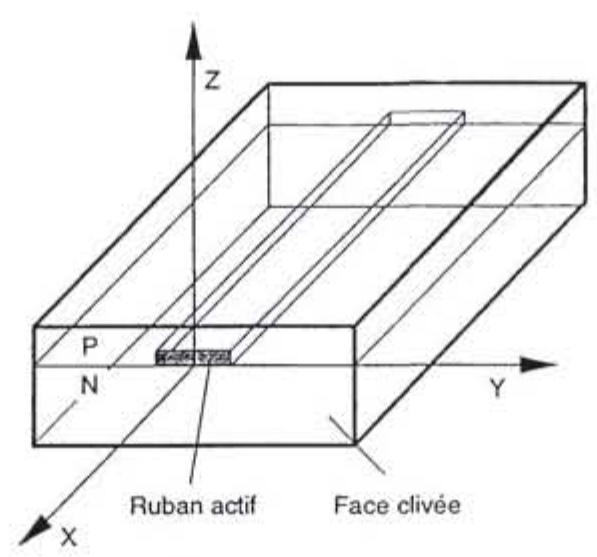

Figure 9 : Géométrie d'une puce de diode Laser 


\section{STUCTURES ET TECHNOLOGIE}

\subsection{Diode Laser PEROT-FABRY à géométrie ruban $[6,7]$}

Une puce de diode Laser de référence est représentée schématiquement sur la figure 9 qui définit les axes utilisés dans la suite. Elle comprend une région active en forme de ruban, en matériau dont le gap détermine la longueur d'onde à émettre, insérée dans une jonction P-N en matériau à bande interdite supérieure de manière à former une structure à double hétérojonction. La lumière se propage selon l'axe $\mathrm{Ox}$ en étant amplifiée par émission stimulée. Elle se réfléchit partiellement sur les faces "avant" et "arrière", qui sont le plus souvent obtenues par clivage du cristal suivant des plans (110), dont le coefficient de réflexion naturel est voisin de 0,3 et qui constituent un interféromètre de PEROT-FABRY. La distance entre les faces clivées, "longueur" du Laser, est de l'ordre de 300 microns.

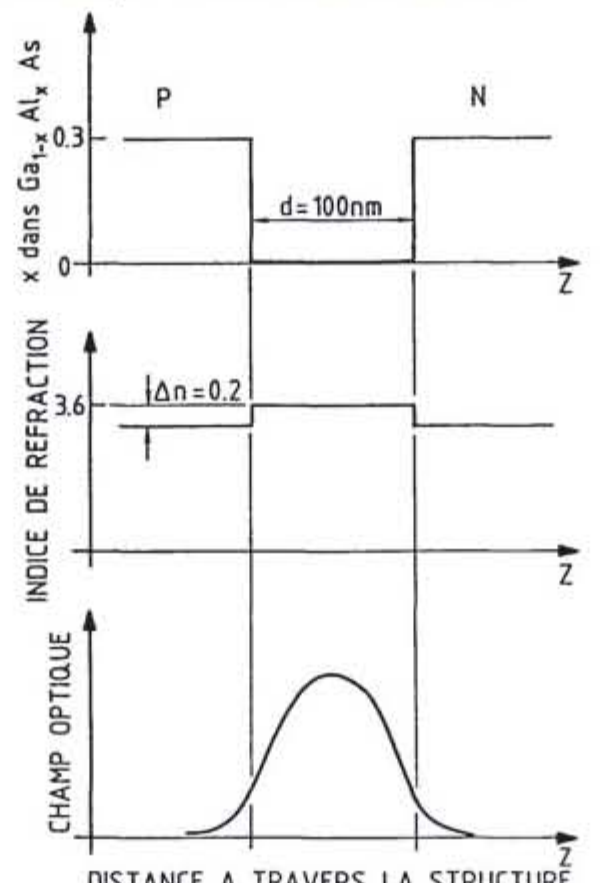

Figure 10 : Profils à travers une double hétéro-jonction du système GaAlAs, de la composition du matériau (10a), de l'indice de réfraction (10b), et de l'onde électromagnétique guidée (10c).

\subsection{Guide plan constitué par une double hétérojonction [6,7]}

La structure à double hétérojonction forme un guide diélectrique assurant un confinement optique suivant la direction $\mathrm{Oz}$ au voisinage de la zone active ainsi que le montre la figure 10 . En effet l'indice de réfraction des semi-conducteurs est de façon générale une fonction décroissante du gap et a le profil indiqué sur la figure pour une structure supposée en GaAlAs. L'étude des modes guidés par une structure symétrique, montre que seul existe, pour une 
épaisseur de zone active assez faible, le mode fondamental dont la distribution de champ optique est également représenté sur la figure 10. Cette configuration est recherchée car elle procure un diagramme de directivité ayant un seul lobe et plus facile à exploiter dans les applications.

On voit également sur la figure que le recouvrement entre le champ optique et la zone active est imparfait : le champ optique s'étend partiellement dans les couches de confinement à indice plus faible. L'efficacité du confinement optique est caractérisée par un facteur de confinement $\Gamma$ défini par la formule suivante :

$$
\Gamma=\frac{\int_{-d / 2}^{+d / 2} E^{2} d z}{\int_{-\infty}^{+\infty} E^{2} d z}
$$

où $\mathrm{d}$ est l'épaisseur de la zone active, voisine en pratique de 0,1 micron, et $\mathrm{E}$ est le champ électrique optique.

\subsection{Guidages transverses [7]}

Alors que l'épaisseur du ruban actif est généralement fixée par une opération d'épitaxie et est uniforme sur la surface du substrat, sa largeur I (direction Oy sur la figure 9) est définie par une étape technologique de microlithographie pour aboutir à un guide bidimensionnel. On distingue deux familles de guidages transverses, susceptibles de nombreuses variantes selon les modes de réalisation.

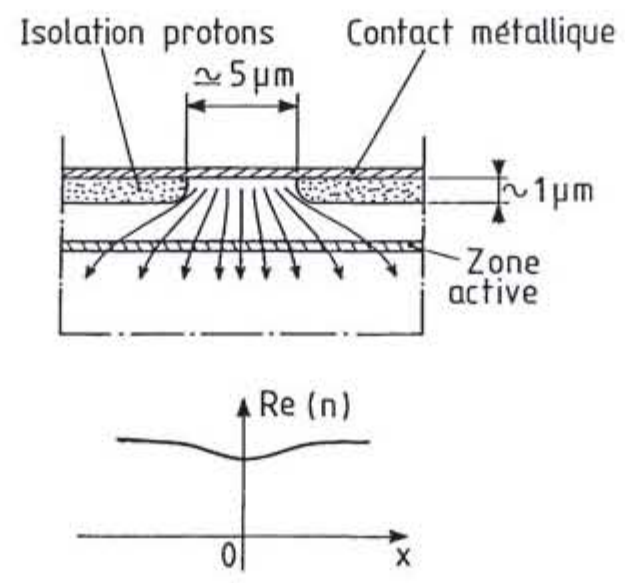

Figure 11 : Guidage par le gain ; structure (11a) et profil de la partie réelle de l'indice (11b)

La figure 11 décrit un exemple de ruban à guidage par le gain, où le matériau de la région active est conservé sur toute la surface du dispositif mais le passage du courant est limité à la région du ruban par une implantation locale de protons qui a pour effet de rendre le matériau isolant. Il en résulte que la densité d'électrons injectés et le gain (représenté par la partie imaginaire de l'indice de réfraction) sont maximaux au centre du ruban alors que la partie réelle de l'indice y présente un minimum. La structure est donc antiguidante ce qui induit des pertes à 
la propagation qui doivent être compensées par le gain. La largeur d'un ruban élémentaire d'une diode Laser à guidage par le gain est de l'ordre de quelques microns afin d'obtenir un fonctionnement stable sur le mode fondamental transverse.
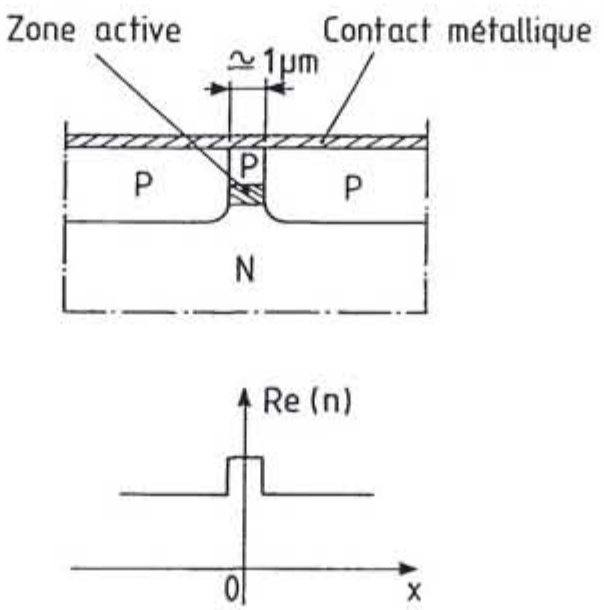

Figure 12 : Guidage par l'indice; structure (12a) et profil de la partie réelle de l'indice (12b).

La figure 12 montre une structure de diode Laser à guidage par l'indice, dite à hétérostructure enterrée, où une opération de microlithographie a enlevé le matériau actif en dehors de la région du ruban, puis une deuxième épitaxie a enterré le ruban dans le matériau constituant la deuxième couche de confinement. Le profil de la partie réelle de l'indice de réfraction selon la direction Oy est alors celui représenté sur la figure, il procure un vrai guidage transverse. De plus, le passage du courant électrique est localisé à la région active car les barrières de potentiel aux homojonctions $\mathrm{P}-\mathrm{N}$ à grande bande interdite situées de part et d'autre sont supérieures à celles de la double hétérojonction. La largeur du ruban d'une diode Laser à guidage par l'indice du type représenté est voisine de un micron afin d'obtenir un fonctionnement sur le mode fondamental suivant la direction $\mathrm{Oy}$.

\section{Fabrication des diodes Laser}

La fabrication des diodes Laser, comme celle des autres composants à semi-conducteur, comporte d'abord une série d'étapes collectives où sont traités plusieurs milliers de composants élémentaires sur un substrat de quelques $\mathrm{cm}^{2}$ :

- une ou plusieurs opérations d'épitaxie,

- diffusion ou implantation éventuelles d'impuretés,

- dépôt de métallisations pour les contacts ohmiques,

- opérations intermédiaires éventuelles de microlithographie, déterminant la géométrie du dispositif dans le plan du substrat, ainsi par exemple le nombre et la largeur des rubans,

- amincissement du substrat à environ 100 microns.

Les composants élémentaires sont alors individualisés comme indiqué sur la figure 13 :

- clivage en barrettes, 
- clivage ou sciage en puces de largeur quelques centaines de microns.
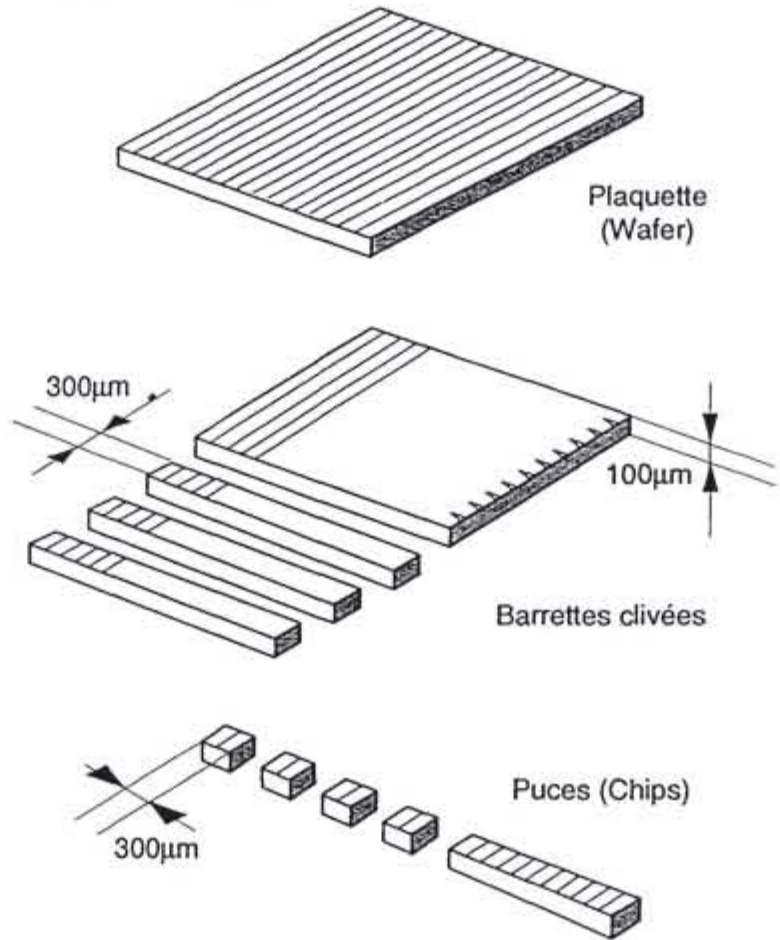

Figure 13 : Découpe par clivage d'une plaque en barrettes puis en puces individualisées.

Enfin est effectuée une série d'opérations individuelles :

- dépôt éventuel de couches diélectriques sur les faces clivées, afin d'en optimiser la réflectivité et/ou les passiver contre l'environnement,

- soudure sur des embases permettant de dissiper la chaleur,

- prise des contacts électriques,

- encapsulation dans des boîtiers dépendant de l'application (sortie du rayonnement par une fenêtre ou par une fibre optique).

\section{CARACTÉRISTIQUES DE FONCTIONNEMENT}

\subsection{Caractéristique I/V et Q/V [4,5]}

Les caractéristiques d'entrée du transducteur électrique-optique qu'est une diode Laser sont celles d'une diode à semi-conducteur polarisée en direct. En régime continu, on a vu précédemment la relation $\mathrm{I} / \mathrm{V}$ pour une diode idéale. Au cas particulier d'une diode Laser deux points sont à préciser.

- Au-delà du seuil défini plus loin, la condition de BERNARD ET DURAFFOURG implique que la tension aux bornes de la jonction est constante et voisine de $\mathrm{E}_{\mathrm{g}} / \mathrm{q}$, par suite sa résistance dynamique est négligeable. 
- Il existe en général des éléments parasites dus à l'environnement de la région active : résistance en série avec la jonction $\mathrm{R}_{\mathrm{S}}$, diode parallèle constituée par les jonctions latérales et laissant passer un courant de fuite que l'on cherche à minimiser.

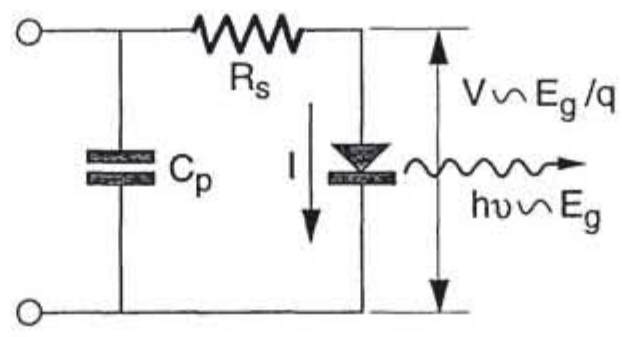

Figure 14 : Schéma électrique équivalent d'une puce de diode Laser

En régime dynamique, il faut considérer également les diverses capacités de la structure, qui peuvent limiter la vitesse de réponse du composant :

- celle de la jonction active, négligeable en général car elle est court-circuitée par la résistance dynamique très faible,

- celle des jonctions parallèles $\mathrm{C}_{\mathrm{p}}$, que l'on diminue par exemple en combinant hétérostructure enterrée et implantation de protons.

On peut finalement donner pour la puce de diode Laser un schéma équivalent simple tel que celui représenté sur la figure 14 .

\subsection{Caractéristique $\mathrm{P} / \mathrm{I}$ en continu $[6,7]$}

Les propriétés de conversion d'énergie électrique en énergie optique du transducteur sont représentées sur la caractéristique $\mathrm{P} / \mathrm{I}$, donnant la puissance optique émise en fonction du courant injecté et dont l'allure générale est représentée sur la figure 15 . On en tire plusieurs paramètres utilisés pour caractériser le composant : courant de seuil $\mathrm{I}_{\mathrm{S}}$, pente de conversion $\mathrm{S}$ ( $\mathrm{W} / \mathrm{A})$, puissance de saturation $\mathrm{P}_{\mathrm{sat}}$, puissance de dégradation catastrophique $\mathrm{P}_{\mathrm{dc}}$.

\subsubsection{Courant de seuil}

La condition de seuil pour un Laser exprime que le gain d'émission stimulée compense les pertes et que le dispositif fonctionne comme un auto-oscillateur aux fréquences optiques.

Le gain du mode guidé ou gain modal est donné par :

$$
\mathrm{g}_{\mathrm{m}}=\Gamma \mathrm{g}
$$

où g est le gain du matériau massif dû aux transitions bande à bande et $\Gamma$ est le facteur de confinement défini précédemment.

Les pertes sont de deux natures :

- pertes nuisibles, réparties, dues à l'absorption et à la diffusion du mode guidé, caractérisées par un coefficient d'absorption modal $\alpha_{\mathrm{m}}\left(\mathrm{cm}^{-1}\right)$, 
- pertes utiles, localisées, dues à la transmission des miroirs partiellement réfléchissants.

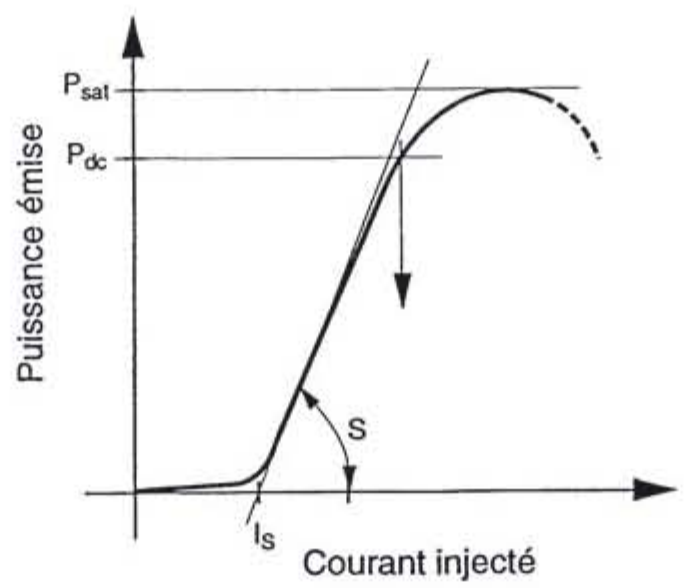

Figure 15 : Caractéristique P/ (Puissance émise / Courant injecté) d'une diode Laser.

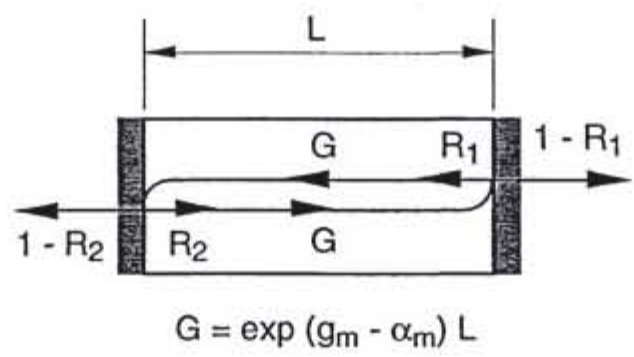

Figure 16 : Schéma latéral d'une diode Laser de type Perot-Fabry.

La condition de seuil s'obtient en écrivant qu'après un aller-retour dans la cavité PEROTFABRY, l'intensité du mode guidé se retrouve identique à elle-même (Fig. 16), soit :

$$
R_{1} R_{2} \exp \left[2\left(g_{m s}-\alpha_{m}\right) L\right]=1
$$

où $R_{1}$ et $R_{2}$ sont les coefficients de réflexion des deux faces clivées, $g_{m s}$ est le gain modal au seuil et $\mathrm{L}$ est la distance entre les deux faces.

Le courant de seuil, paramètre plus utile pour une diode, peut être évalué en remarquant qu'au seuil, la recombinaison stimulée est encore négligeable. Par suite le courant injecté compense exactement la recombinaison radiative spontanée (en supposant négligeable la recombinaison non-radiative). On a done :

$$
I_{S}=q B n_{s}^{2} L I d
$$


où $\mathrm{n}_{\mathrm{S}}$ est la densité d'électrons au seuil, voisine de $10^{18} \mathrm{~cm}^{-3}$ (figure 4). On remarque que le produit ( $\mathrm{Lld}$ ) est le volume de la région active. Pour des composants standards, $\mathrm{n}_{\mathrm{S}}$ est voisin de $\mathrm{n}_{\mathrm{t}}$ défini précédemment (figure 4), et le produit $\mathrm{q} \mathrm{B} \mathrm{n}_{\mathrm{s}}^{2}$ vaut $1,610^{7} \mathrm{~A} / \mathrm{cm}^{3}$.

\subsubsection{Rendement différentiel}

L'efficacité du dispositif peut être caractérisée par la pente de conversion en W/A ou, de façon équivalente, par le rendement quantique différentiel externe $\eta_{\mathrm{e}}$, rapport du nombre de photons émis au nombre d'électrons injectés. On a en effet :

$$
\mathrm{S}=(\mathrm{h} v / \mathrm{q}) \eta_{\mathrm{e}}
$$

En dessous du seuil, l'émission est essentiellement spontanée et, même si le rendement quantique interne $\eta_{\mathrm{i}}$ est élevé, très peu de photons sont émis dans le mode du guide que l'on utilise, par suite $\eta_{\mathrm{e}}$ est très faible. Au-dessus du seuil, d'une part $\eta_{\mathrm{i}}$ est voisin de $100 \%$ car l'émission est essentiellement stimulée et la durée de vie radiative est très faible, et d'autre part le rendement de sortie des photons $\eta_{0}$ est élevé. Il est en effet donné par le rapport entre les pertes utiles aux faces clivées et la somme des pertes utiles et des pertes nuisibles de sorte que l'on a :

$$
\eta_{\mathrm{o}}=1 /\left[1+2 \alpha_{\mathrm{m}} \mathrm{L} / \log \left(1 / \mathrm{R}_{1} \mathrm{R}_{2}\right)\right]
$$

Compte tenu des valeurs typiques des paramètres mis en jeu, $\eta_{\mathrm{e}}=\eta_{\mathrm{i}} \eta_{\mathrm{o}}$ peut être compris entre 20 et $80 \%$ pour les diodes Laser PEROT-FABRY considérées.

Par ailleurs il peut être intéressant d'ajuster indépendamment les réflectivités des faces "avant" et "arrière" du dispositif en vue d'une application donnée. On peut ainsi donner une réflectivité de 1 à la face "arrière" afin de faire sortir toute l'énergie par la face "avant". On peut montrer simplement que l'on a alors :

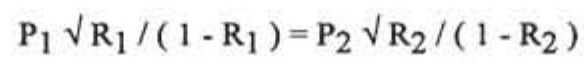

\subsubsection{Limites à la puissance émise}

La dégradation catastrophique [6,7] (COD ou Catastrophic Optical Degradation) est un processus irréversible, dû à l'échauffement local des faces clivées allant jusqu'à la fusion, sous les effets conjoints de l'absorption du flux optique et de la recombinaison non-radiative de surface. La densité de flux optique correspondante dépend dans le détail de la structure du composant, de la passivation des faces et du régime de fonctionnement. En régime continu, elle est comprise entre $10^{5}$ et $10^{6} \mathrm{~W} / \mathrm{cm}^{2}$ pour les composants à base de GaAlAs, elle est plus élevée pour un fonctionnement en impulsions courtes $(<1 \mu \mathrm{s})$. Elle n'est pratiquement jamais atteinte pour les composants à base de GaInAsP.

La saturation de la caractéristique $\mathrm{P} / \mathrm{I}$ est au contraire un processus réversible, dû principalement à l'échauffement général de la jonction. En effet le courant de seuil est une fonction rapidement croissante de la température de jonction $\mathrm{T}_{\mathrm{j}}$, caractérisée par le paramètre empirique $T_{0}$ ayant la dimension d'une température et défini par :

$$
I_{S}=I_{r} \exp \left[\left(T_{j}-T_{r}\right) / T_{0}\right]
$$

où $\mathrm{I}_{\mathrm{r}}$ est le courant de seuil à la température de référence $\mathrm{T}_{\mathrm{r}}$. 
On caractérise habituellement l'échauffement par une résistance thermique $R_{\text {th }}(\mathrm{K} / \mathrm{W})$ définie par :

$$
T_{j}-T_{s}=R_{t h} V I
$$

où $\mathrm{T}_{\mathrm{S}}$ est la température du support.

Ainsi lorsque l'on augmente le courant d'injection, le courant de seuil augmente également et si $I_{S}$ augmente plus vite que $I$ la puissance émise décroît, d'oủ la saturation montrée sur la figure 16. La valeur de $\mathrm{R}$ th dépend considérablement de la géométrie du composant (surface active) et de la technologie de montage (dissipateur thermique), alors que $\mathrm{T}_{0}$ est principalement une caractéristique des matériaux semi-conducteurs employés :

- GaAlAs, $\mathrm{T}_{0} \sim 150 \mathrm{~K}$,

- GaInAsP, $\mathrm{T}_{0} \sim 70 \mathrm{~K}$.

Il en résulte que cet effet est beaucoup plus marqué pour les composants à base de InP.

\subsection{Caractéristiques dynamiques $[7,8]$}

Les diodes Laser sont très utilisées comme convertisseurs de signaux du domaine électronique, bien adapté au traitement et au stockage des informations, au domaine optique (ou photonique), bien adapté à leur transmission. Leur intérêt dans ces fonctions vient de ce que la modulation de la porteuse optique par les signaux électriques peut être effectuée par le courant injecté. Cette technique de modulation directe, à distinguer de la modulation externe où le signal agit sur la porteuse dans un composant modulateur spécifique, est plus économique en coût de composants comme de puissance de commande. En plus des limitations extrinsèques à la vitesse de modulation, dues aux éléments parasites de circuit schématisés sur la figure 15 , il y a lieu d'examiner les limitations intrinsèques aux réponses temporelle ou fréquentielle en fonction du type de modulation retenu.

\subsubsection{Réponse temporelle}

La réponse d'une diode Laser à un échelon de courant $\mathrm{I}>\mathrm{I}_{\mathrm{S}}$ injecté à $\mathrm{t}=0$ est représentée sur la figure 17. On remarque d'abord un retard à l'émission stimulée $t_{d}$, temps nécessaire pour que la densité d'électrons hors équilibre $\mathrm{n}$ passe de 0 à la valeur de seuil $\mathrm{n}_{\mathrm{S}}$. Un calcul simple, dans l'approximation de la durée de vie, montre que

$$
t_{d}=\tau \log \left(I /\left(I-I_{S}\right)\right)
$$

de sorte que $t_{d}$ a l'ordre de grandeur de la durée de vie totale. Pour $I=1,58 I_{S}, t_{d}$ vaut exactement $\tau$ puis décroit lentement lorsque 1 augmente. Ce retard, gênant en modulation d'impulsions à haut débit, peut être annulé en prépolarisant le composant au seuil par un courant continu.

L'émission stimulée montre ensuite un comportement résonnant amorti, dont les caractéristiques, fréquence et temps d'amortissement, dépendent dans le détail du point de fonctionnement utilisé. Ce comportement a pour origine physique les échanges entre les énergies stockées dans la région active sous forme électronique et sous forme optique et limite actuellement la vitesse de modulation vers 20 Gbauds. 


\subsubsection{Réponse fréquentielle intrinsèque}

La figure 18 montre les variations de la puissance optique émise en fonction de la fréquence pour un courant de modulation sinusoïdal d'amplitude petite par rapport au courant continu de polarisation, supérieur au seuil, auquel il est superposé. Les courbes présentées résultent d'un modèle linéarisé intégrant deux équations différentielles couplées décrivant les évolutions des populations d'électrons et de photons.

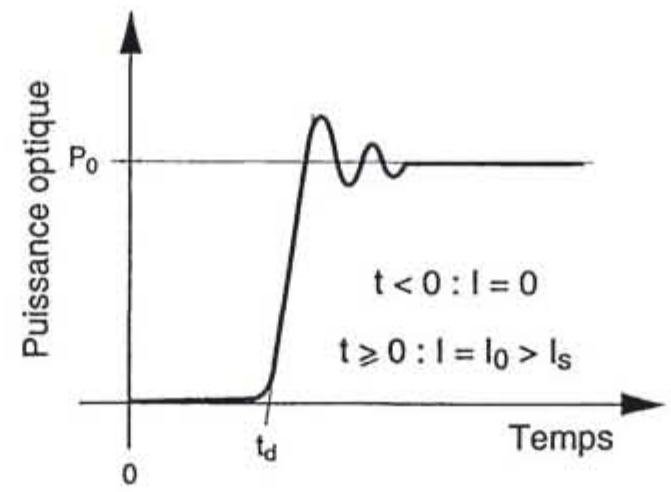

Figure 17 : Réponse temporelle d'une diode Laser à un échelon de courant supérieur au courant de seuil.

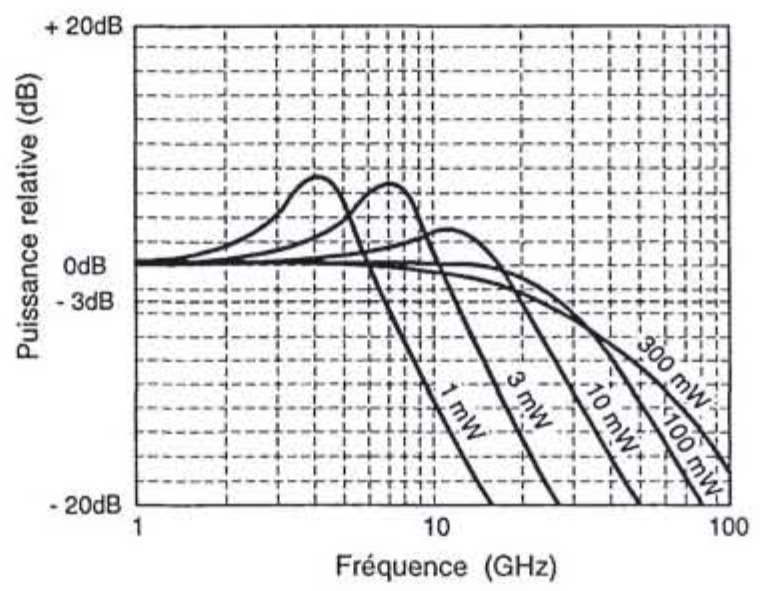

Figure 18 : Réponse fréquentielle d'une diode Laser en petits signaux.

On retrouve sur ces courbes le comportement résonnant de la figure 17, et l'on voit de plus l'effet du choix de point de fonctionnement, caractérisé par la puissance moyenne émise $\mathrm{P}_{0}$, sur la fréquence de résonance et sur l'amortissement. La modélisation montre et les expériences confirment que la fréquence de résonance croit en $\sqrt{\mathrm{P}_{0}}$ alors que l'amortissement croit comme 
$\mathrm{P}_{0}$. Il en résulte que la bande passante mesurée par exemple à $-3 \mathrm{~dB}$ passe par un maximum dépendant essentiellement du matériau et dont l'ordre de grandeur est de $30 \mathrm{GHz}$.

\subsubsection{Fonctionnement en modes bloqués}

Certaines structures de diodes Laser comprenant une section jouant le rôle d'absorbant saturable passif ou commandé électriquement permettent d'obtenir un fonctionnement en modes bloqués, libre ou synchronisé, délivrant des impulsions courtes (sub-ps) avec des fréquences de répétition supérieures à $100 \mathrm{GHz}[9,10]$.

\subsubsection{Bruit d'émission [8]}

Le bruit à l'émission d'une diode Laser a pour origine physique l'émission spontanée s'ajoutant aléatoirement en phase avec l'émission stimulée. Les fluctuations résultantes de la puissance émise limitent la dynamique de la transmission de signaux analogiques et sont habituellement caractérisées après détection par le RIN (Relative Intensity Noise), mesurant l'excès de bruit par rapport au bruit de grenaille :

$$
\mathrm{RIN}=\left(\left\langle\mathrm{I}^{2}\right\rangle / \mathrm{B}-2 \mathrm{qI}\right) / \mathrm{I}^{2}
$$

où $\left\langle\mathrm{I}_{\mathrm{b}}^{2}\right\rangle$ est la valeur quadratique moyenne du photocourant de bruit, B est la bande passante de la mesure et $I$ est le courant continu détecté.

Le RIN se mesure en principe en secondes (dimension de l'inverse d'une fréquence), mais est en fait souvent et incorrectement donné en $\mathrm{dB} / \mathrm{Hz}$, la bande passante de la mesure étant supposée de $1 \mathrm{~Hz}$. De façon générale, il décroît quand $\mathrm{P}_{0}$ augmente et dépend de la fréquence avec un maximum à la fréquence de résonance. Sa valeur peut atteindre $10^{-16} \mathrm{~s}$, soit un RIN de $160 \mathrm{~dB} / \mathrm{Hz}$.

\subsection{Caractéristiques spectrales [6,7]}

\subsubsection{Caractéristique $P / \lambda$}

L'une des caractéristiques de sortie de la diode Laser est la distribution de la puissance optique émise en fonction de la longueur d'onde ou spectre d'émission.

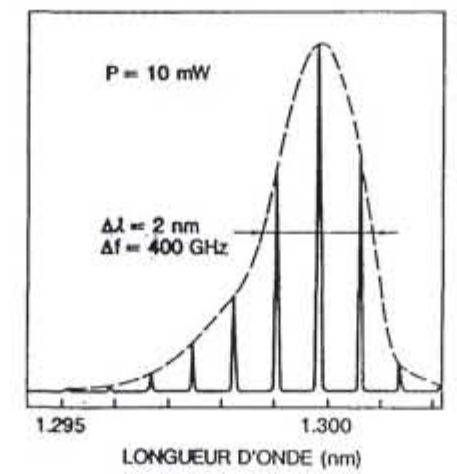

Figure 19 : Spectre d'émission d'une diode Laser Perot-Fabry pour télécommunications. 
Un exemple typique relatif à une diode PEROT-FABRY est représenté sur la figure 19. La longueur d'onde moyenne (1300 nm dans l'exemple considéré) est fixée par la composition du matériau de la région active et plusieurs raies apparaissent dans une enveloppe de largeur à mi-hauteur $\Delta \lambda$ de 2 à $3 \mathrm{~nm}$. En effet la condition de seuil écrite plus haut est incomplète, il est également nécessaire d'exprimer que la phase de l'onde guidée se retrouve identique après un aller-retour dans la cavité soit :

$$
2 \mathrm{Ln} / \lambda_{\mathrm{k}}=\mathrm{k}
$$

où $\mathrm{n}$ est l'indice de réfraction modal, proche de l'indice de réfraction des matériaux constituant le guide optique et $\lambda_{k}$ est la longueur d'onde dans le vide satisfaisant l'équation pour le nombre entier $\mathrm{k}$.

Plusieurs $\lambda_{\mathrm{k}}$ se trouvent en général situées dans l'enveloppe oủ est satisfaite la condition sur l'intensité du mode guidé et donnent les positions des raies observées ou modes longitudinaux de la cavité. Les diodes Laser PEROT-FABRY sont donc appelées parfois diodes Laser multimodes.

L'écart $\delta \lambda$ entre deux modes consécutifs $(\delta k=1)$ peut être obtenu simplement à partir de l'équation précédente et en tenant compte des variations de $\mathrm{n}$ avec $\lambda$ :

$$
\delta \lambda=\lambda^{2} /(2 \mathrm{~L}(\mathrm{n}-\lambda \mathrm{dn} / \mathrm{d} \lambda))
$$

Cet écart est de 0,3 à $1 \mathrm{~nm}$ pour les diodes Laser de référence

\subsubsection{Réglage de longueur d'onde}

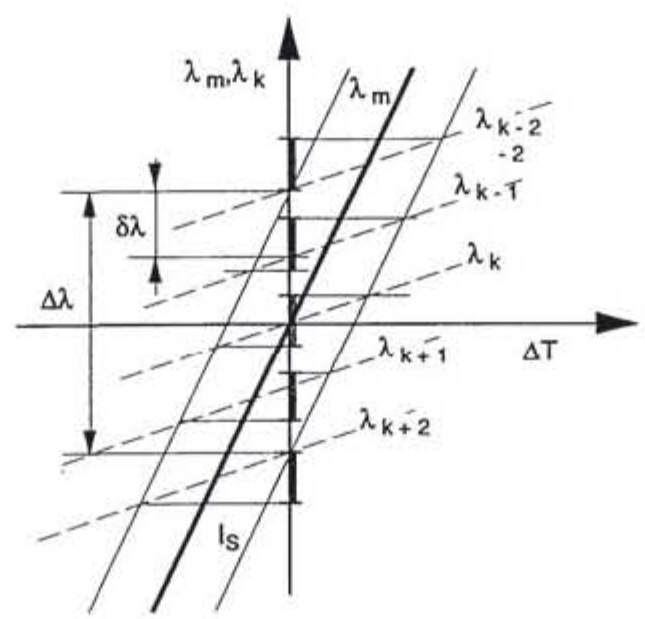

Figure 20 : Réglage de la longueur d'onde d'une diode Laser par la température.

Il est intéressant pour de nombreuses applications de disposer d'une source cohérente à longueur d'onde ajustable, ainsi la température est un paramètre permettant simplement de commander la longueur d'onde d'émission des diodes Laser. En effet un accroissement de T a pour effet de diminuer le gap des semi-conducteurs considérés et par suite d'augmenter la longueur d'onde moyenne du spectre d'émission. La vitesse d'augmentation $\mathrm{d} \lambda_{\mathrm{m}} / \mathrm{dT}$ est voisine de $0,5 \mathrm{~nm} / \mathrm{K}$ pour les matériaux de la famille $\mathrm{InP}$ à la température de $300 \mathrm{~K}$. 
Dans l'approximation oủ la relation liant l'indice à la température est de la forme

$$
\mathrm{n}(\lambda, \mathrm{T})=\mathrm{n}\left(\lambda-\lambda_{\mathrm{m}}(\mathrm{T})\right)
$$

on peut montrer que la longueur d'onde des modes varie avec $\mathrm{T}$ de la façon suivante :

$$
\mathrm{d} \lambda_{\mathrm{k}} / \mathrm{dT}=\mathrm{d} \lambda_{\mathrm{m}} / \mathrm{dT}(\lambda \mathrm{dn} / \mathrm{d} \lambda) /(\mathrm{n}-\lambda \mathrm{dn} / \mathrm{d} \lambda)
$$

La vitesse d'augmentation de la longueur d'onde des modes est alors voisine de $0,1 \mathrm{~nm} / \mathrm{K}$ dans les mêmes conditions.

L'analyse des variations avec la température de $\lambda_{\mathrm{m}}$ et des $\lambda_{\mathrm{k}}$ portées sur le même graphique (Fig. 20) permet de tirer les conclusions suivantes pour le composant considéré :

- Le domaine de longueur d'onde couvert par un mode est seulement d'une fraction de $\Delta \lambda$, ici $0,5 \mathrm{~nm}$ environ.

- Le domaine de longueur d'onde couvert par l'ensemble des modes n'est pas continu.

\subsection{Diagramme de directivité : caractéristiques $P / \theta[6,7\}$}

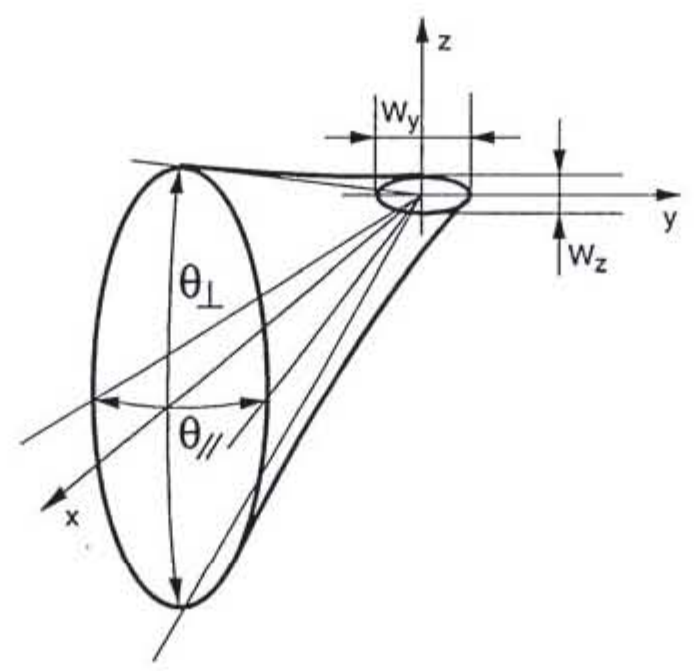

Figure 21 : Diagramme de champ lointain d'une diode Laser

La distribution en amplitude et en phase du champ électromagnétique sur la face de sortie, ou champ proche, détermine le diagramme de directivité, ou champ lointain, caractérisé par les largeurs à mi-hauteur du faisceau émis $\theta_{\perp}$ et $\theta_{/ /}$(Fig. 21). La diffraction fixe ces angles par les relations approximatives suivantes :

$$
\theta_{\perp} \approx \lambda / \mathrm{w}_{\mathrm{z}} \quad \theta_{/ /} \approx \lambda / \mathrm{w}_{\mathrm{y}}
$$

où $\theta_{\perp}$ et $\theta_{/ /}$sont les angles dans les plans respectivement perpendiculaire $(\mathrm{xOz})$ et parallèle $(\mathrm{xOy})$ au substrat, tandis que $\mathrm{w}_{\mathrm{x}}$ et $\mathrm{w}_{\mathrm{y}}$ sont les dimensions (taille de faisceau) du champ proche dans le plan de la facette de sortie. $\theta_{\perp}$ et $\theta_{/ /}$sont en général différents et 
typiquement compris entre $20^{\circ}$ et $60^{\circ}$.

\section{DIODES LASER À PUITS QUANTIQUES}

\subsection{Structures à confinements séparés}

On a vu qu'en première approximation le courant de seuil est proportionnel au volume de la région active d'une diode Laser. La disponibilité dans les années 80 de techniques d'épitaxie permettant de contrôler l'épaisseur de couches épitaxiales à une couche atomique près, soit $0,25 \mathrm{~nm}$, a donc soulevé de façon concrète la question de la valeur minimale atteignable pour la densité de courant de seuil $\mathrm{J}_{\mathbf{S}}$.

L'analyse de la propagation guidée dans la double hétérostructure de référence décrite sur la figure 10 montre alors que pour une épaisseur de couche active $d<100 \mathrm{~nm}$ le facteur de confinement $\Gamma$ varie comme $d^{2}$ et devient donc très petit. Or, bien que le gain modal au seuil $\mathrm{g}_{\mathrm{ms}}$ dépende peu de la structure des couches épitaxiales, le gain du matériau au seuil $\mathrm{g}_{\mathrm{S}}=\mathrm{g}_{\mathrm{ms}} / \Gamma$ augmente très vite si $d$ décroît et par suite $\mathrm{J}_{\mathrm{S}}$ passe par un minimum d'environ $1000 \mathrm{~A} / \mathrm{cm}^{2}$ pour $\mathrm{d}$ voisin de $100 \mathrm{~nm}$.

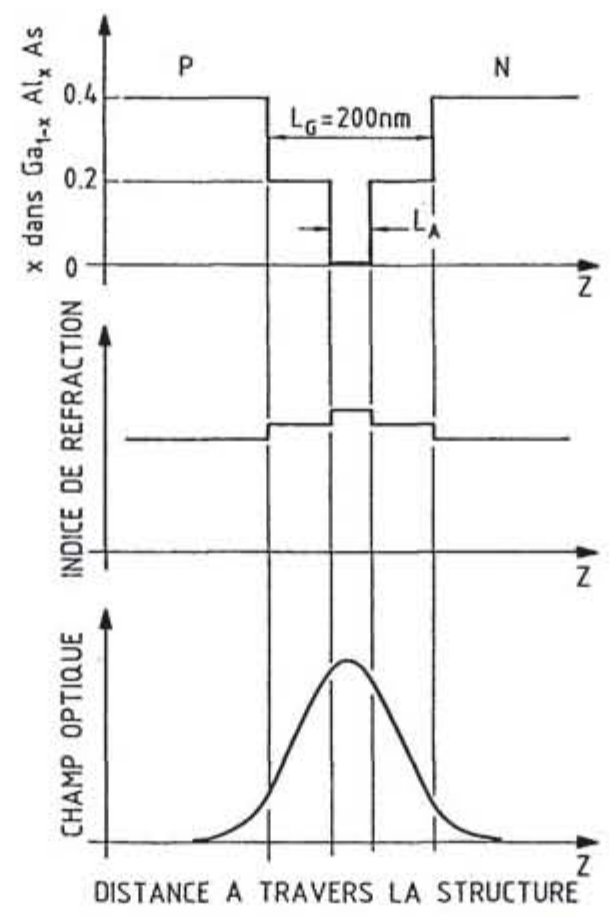

Figure 22: Structure SCH-SQW (Separate Confinement Heterostructure-Single Quantum Well.

Afin de dépasser cette limite, THOMSON [11] a proposé l'utilisation de structures dites à confinements séparés, ou SCH (Separate Confinement Heterostructure), où les confinements 
électrique (couche active) et optique (guide) sont assurées par des couches différentes comme représenté sur la figure 22 . La couche active d'épaisseur $\mathrm{L}_{\mathrm{A}}$ et supposée en GaAs est insérée dans un guide optique en $\mathrm{Ga}_{0,8} \mathrm{Al}_{0,2}$ As d'épaisseur $\mathrm{L}_{\mathrm{G}}$ compris entre deux couches de types $\mathrm{P}$ et $\mathrm{N}$ en $\mathrm{Ga}_{0,6} \mathrm{Al}_{0,4} \mathrm{As}$. $\Gamma$ varie alors comme $\mathrm{L}_{\mathrm{A}}$ de sorte que le minimum de $\mathrm{J}_{\mathrm{S}}$ peut atteindre $100 \mathrm{~A} / \mathrm{cm}^{2}$ pour des cavités longues $(>1 \mathrm{~mm})$.

\subsection{Efrets de taille quantique [12]}

La couche active des structures $\mathrm{DH}$ ou SCH constitue un puits de potentiel pour les électrons et pour les trous, compte tenu du raccordement des BC et BV des matériaux des familles GaAlAs ou GaInAsP. Lorsque son épaisseur devient inférieure à leur Libre Parcours Moyen, soit environ $100 \mathrm{~nm}$ à $300 \mathrm{~K}$, de nouveaux effets dits de taille quantique ou bidimensionnels surviennent, et les diodes Laser sont dites à puits quantiques ou QW (Quantum Well). On parle de diodes Laser à puits quantique unique (SQW) ou multiples (MQW) suivant qu'un ou plusieurs puits sont insérés dans le guide optique.

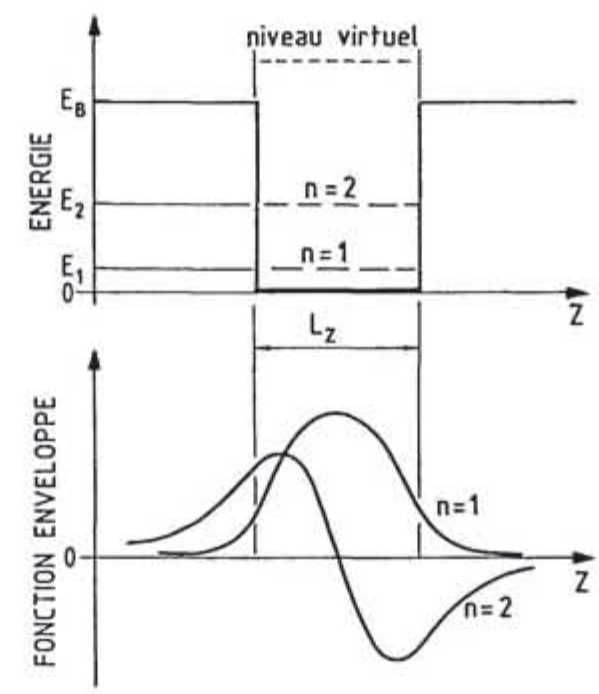

Figure 23 : Fonctions d'onde électroniques dans un puits quantique

Il faut en effet, dans ces conditions, tenir compte de la nature ondulatoire des particules, électrons et trous, les ondes associées étant susceptibles d'interférer avec elles-mêmes après réflexion sur les barrières de confinement. Un puits quantique se comporte donc de façon analogue à un guide optique : seules certaines longueurs d'onde de DE BROGLIE sont permises et caractérisent les états quantiques permis auxquels on peut associer des niveaux d'énergie. La figure 23 montre ainsi l'allure de la fonction d'onde pour les deux premiers états quantiques, numérotés $n=1$ et $n=2$, ainsi que les niveaux d'énergie associés $E_{1}$ et $E_{2}$ dans un puits d'épaisseur $\mathrm{L}_{\mathrm{Z}}$ confiné par des barrières de hauteur $\mathrm{E}_{\mathrm{B}}$. Dans les structures $\mathrm{SCH}$, le 
mouvement des particules est quantifié seulement dans la direction $\mathrm{z}$, elles sont libres dans les directions $x$ et $y$, d'oủ le nom de gaz d'électrons bidimensionnel. La composante $k_{z}=2 \pi / \lambda_{2}$ du vecteur d'onde est quantifiée et, pour un puits infini, bonne approximation pour le premier niveau d'un puits pas trop étroit, on a :

$$
2 \mathrm{~L}_{\mathrm{z}} / \lambda_{\mathrm{zn}}=\mathrm{n}
$$

où $\mathrm{n}$ est un nombre entier et $\lambda_{\mathrm{zn}}$ est la longueur d'onde correspondante.

Rappelons que la relation de DE BROGLIE associe la longueur d'onde à la quantité de mouvement par :

$$
\mathrm{p}_{\mathrm{z}}=\mathrm{h} / \lambda_{\mathrm{z}}
$$

où $\mathrm{p}_{\mathrm{z}}$ est la composante suivant $\mathrm{Oz}$ du vecteur quantité de mouvement de l'électron. L'énergie de l'électron est alors :

$$
E=p^{2} / 2 m_{e}=\left(p_{x}^{2}+p_{y}^{2}+p_{z}^{2}\right) / 2 m_{e}
$$

oủ $\mathrm{m}_{\mathrm{e}}$ est la masse effective de l'électron, de sorte que l'énergie minimale des électrons au dessus du bas de la bande de conduction $\left(p_{x}=p_{y}=0, n=1\right)$ est :

$$
\mathrm{E}_{\mathrm{C} 1}=\mathrm{h}^{2} /\left(8 \mathrm{~m}_{\mathrm{e}} \mathrm{L}_{\mathrm{z}}^{2}\right)
$$

Il existe une relation analogue pour les trous et finalement la structure de bande dans le puits peut être représentée sur la figure 24 où l'on voit que le gap effectif du matériau dans le puits $E_{G}^{\prime}$ a augmenté par rapport à celui du matériau massif $\mathrm{E}_{\mathrm{G}}$. Il est donc possible par un choix convenable de $\mathrm{L}_{\mathrm{z}}$, d'ajuster la longueur d'onde d'émission d'une diode Laser, à composition de matériau actif fixée.

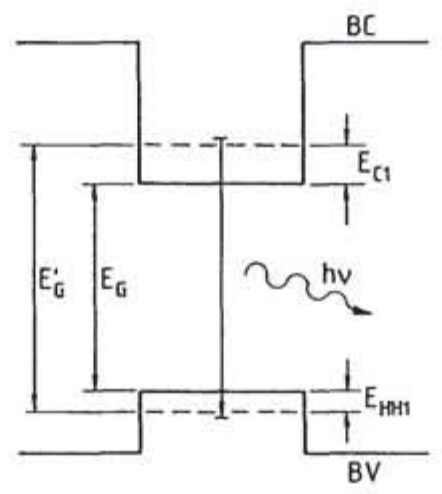

Figure 24 : Gap effectif d'une structure à puits quantique.

L'épaisseur des puits quantiques étant souvent inférieure à l'épaisseur critique en épitaxie (cf. paragraphe 3.2), il est possible d'étendre la gamme des matériaux disponibles aux matériaux désadaptés par rapport au substrat. Outre l'avantage d'accroître le domaine des longueurs d'onde accessibles, l'utilisation de couches pseudomorphiques modifie la structure de 
bande et peut contribuer à diminuer Js et à améliorer certaines propriétés dynamiques des composants.

De façon générale, l'utilisation de structures à confinements séparés et à puits quantiques simples ou multiples (structures SCH-SQW ou SCH-MQW), en offrant plus de paramètres de conception pour les diodes Laser, permet d'accroître le domaine des performances accessibles et pratiquement tous les progrès démontrés récemment ont été obtenus avec de telles structures.

\subsection{Etapes suivantes : fils et boîtes quantiques}

Les techniques modernes d'épitaxie permettent la réalisation industrielle des structures à puits quantique où le mouvement des électrons est quantifié suivant une direction $(\mathrm{Oz}$ sur les figures précédentes). On peut concevoir également des structures où ce mouvement est quantifié suivant deux directions (par exemple $\mathrm{Oy}$ et $\mathrm{Oz}$ ) dans des fils quantiques, ou dans les trois directions de l'espace dans des boîtes quantiques. Le comportement des électrons dans ces structures est alors respectivement unidimensionnel et zéro-dimensionnel [12].

Une réduction supplémentaire du courant de seuil et de sa dépendance en température est attendue des structures de diodes Laser à fils et à boîtes quantiques. Cependant leur réalisation nécessite la définition de leurs caractéristiques géométriques dans le plan xOy avec la même précision que suivant $\mathrm{Oz}$, problème technologique difficile qui en fait encore des objets de laboratoire.

\section{DIVERSIFICATION DES DIODES LASER}

\subsection{Diodes Laser à cavité verticale et microcavités}

Les diodes Laser de référence ont une cavité optique dont l'axe (Ox sur la figure 9) est dans le plan "horizontal" des couches épitaxiales. Au contraire, une diode Laser à cavité verticale (VCSEL, ou Vertical Cavity Surface Emitting Laser) a une cavité optique perpendiculaire à ce plan comme indiqué sur la figure 25 et émet à travers la surface du dispositif, éventuellement à travers son substrat lorsqu'il est transparent à la longueur d'onde considérée. Plusieurs avantages ou possibilités nouvelles sont attendues d'une telle géométrie :

- Réduction du courant de seuil attendue jusqu'à environ $10 \mu \mathrm{A}$ due à la diminution du volume actif.

- Faible coût grâce à la densité élevée de composants par $\mathrm{cm}^{2}$ de substrat, à l'utilisation aisée du rayonnement émis et à la simplification des tests.

- Organisation possible en matrices à deux dimensions et éventuellement adressables.

La principale difficulté de réalisation provient de la faible dimension du matériau actif dans le sens de propagation du rayonnement (épaisseur d'une ou de quelques couches épitaxiales/puits quantiques) qui limite le gain atteignable et rend nécessaire l'emploi de miroirs à coefficient de réflexion très élevé, de préférence conducteurs, et dont l'un au moins doit être partiellement transparent. Ce résultat est atteint le plus souvent au moyen de réflecteurs de BRAGG, comme schématisé sur la figure 25 , constitués d'un empilement de couches épitaxiales de compositions et d'indices de réfraction alternés, par exemple GaAs et AlAs, et d'épaisseur optique $\lambda / 2$. Il est ainsi possible d'atteindre des coefficients de réflexion voisins de $99 \%$ avec 10 à 30 paires de couches.

Les résultats les plus solides sur ce type de composant ont été obtenus avec des structures à puits quantiques contraints en GaInAs, émettant vers $980 \mathrm{~nm}$ où le substrat GaAs est 
transparent [13]. Les recherches visent à démontrer les courants de seuil les plus faibles possibles en diminuant les dimensions transverses, des courants de seuil inférieurs à $1 \mathrm{~mA}$ associés à des puissances en continu voisines de $1 \mathrm{~mW}$ ont été rapportés [14]. A l'inverse, il est possible d'obtenir des puissances cohérentes relativement élevées $(300 \mathrm{~mW})$ par mise en phase de réseaux bidimensionnels de VCSEL [15]. Enfin les autres familles de matériaux susceptibles d'émettre aux longueurs d'onde supérieure $(1300 \mathrm{~nm},[16])$ et inférieure $(670 \mathrm{~nm},[17])$ ont également été utilisées avec succès.

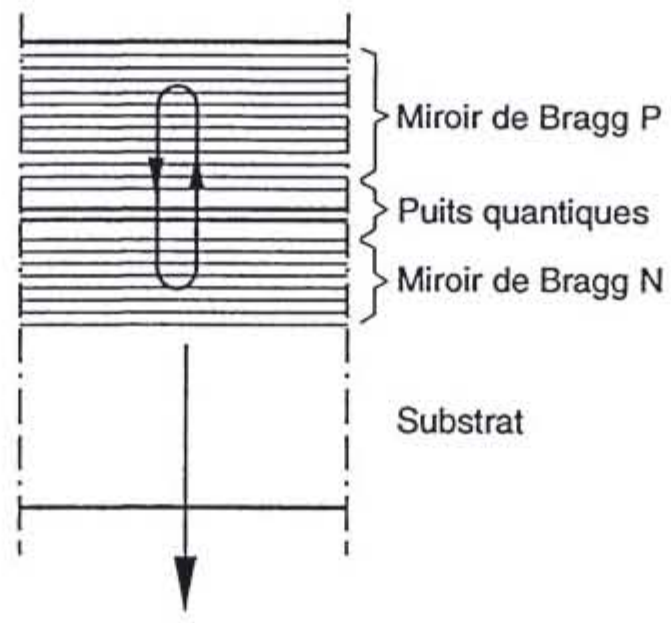

Figure 25 : Diode Laser à émission par la surface et à cavité verticale.

La cavité optique des diodes Laser à cavité verticale peut avoir une "longueur" de l'ordre de grandeur de la longueur d'onde de la lumière émise et en conséquence ne présenter que quelques modes longitudinaux. La réduction des dimensions transverses permettrait une réduction corrélative du nombre de modes transverses et résulter dans un Laser à très peu de modes, voire un seul. L'électrodynamique quantique montre alors que l'émission spontanée dans une telle microcavité est déterminée par les caractéristiques spécifiques de ce mode et peut être renforcée ou diminuée [18]. Il devient alors théoriquement possible de concevoir une diode Laser à seuil nul. L'exploitation de cette possibilité nécessite cependant la réunion difficile de plusieurs conditions :

- Confinement optique dans les trois dimensions.

- Largeur spectrale voisine de celle de la cavité.

- Recombinaison non radiative en volume et en surface négligeable.

- Injection électrique efficace.

- Bonne dissipation thermique.

Les démonstrations actuelles de principe utilisent le plus souvent le pompage optique à basse température [19] ou des stuctures de diodes électroluminescentes "à cavité résonnante" (RCLED) dont les performances en vitesse de réponse et en largeur spectrale sont améliorées [20]. 


\subsection{Diodes Laser de puissance}

\subsubsection{Conception des dispositifs de puissance $[21,22]$}

Que la puissance maximale émise par une diode Laser soit fixée par la saturation thermique ou par la dégradation catastrophique, le principal moyen de l'accroître est d'augmenter la largeur totale de la région active de façon à augmenter la surface émissive et à diminuer la résistance thermique.

Ce résultat peut être obtenu le plus simplement par la réalisation de rubans "larges", par exemple $100 \mu \mathrm{m}$, mais on est limité dans cette voie par l'apparition de modes parasites de la cavité et par des phénomènes de filamentation. Il peut également être obtenu par l'intégration monolithique en parallèle de plusieurs diodes Laser élémentaires à ruban étroit constituant une diode Laser en réseau, puis de plusieurs réseaux constituant une barrette semblable à celles de la figure 13 dont la largeur peut aller jusqu'à $1 \mathrm{~cm}$ et comprenant jusqu'à 1000 rubans élémentaires.

Ces barrettes constituent des émetteurs linéaires qu'il est possible d'assembler pour constituer des émetteurs surfaciques, de façon hybride par empilement (stacks, Fig. 26a) ou de façon monolithique (plaques, Fig. 26b). Dans ce dernier cas, il est nécessaire d'intégrer aussi des dispositifs renvoyant le rayonnement émis perpendiculairenent au plan de la plaque, miroir à $45^{\circ}$ ou réseau de diffraction.

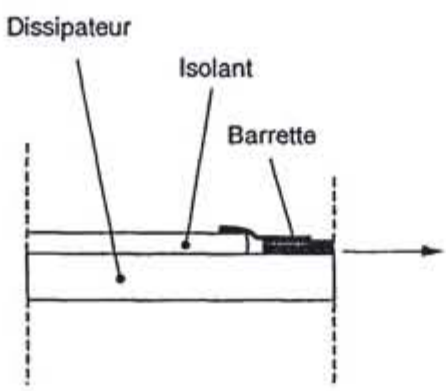

a) Empilement (Stack) Hybride

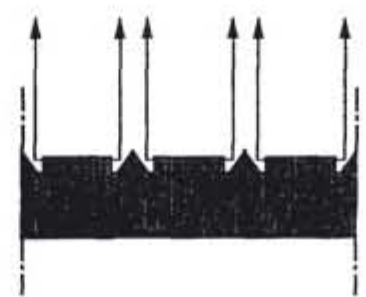

b) Plaque $2 D$ Monolithique

Figure 26 : Module pour empilement (26a) et plaque monolithique (26b).

\subsubsection{Régime de fonctionnement}

L'architecture optimale d'un composant de puissance, en particulier son support assurant l'évacuation de la chaleur dissipée, dépend de plus du régime de fonctionnement nécessaire dans l'application considérée :

- contimu $(\mathrm{CW})$ où les effets thermiques sont importants;

- pulsé où la durée des impulsions (typiquement inférieure à $1 \mu \mathrm{s}$ ) et leur fréquence de répétition sont tels que les échauffements transitoire et moyen sont négligeables et la dégradation des faces limite la puissance;

- quasi contimu (QCW) en impulsions longues (durée typiquement supérieure à $100 \mu \mathrm{s}$, régime concernant essentiellement l'application au pompage de Lasers à solides), où le 
mécanisme limitant dépend de la fréquence de répétition des impulsions.

\subsubsection{Recherche de la puissance cohérente}

Les structures précédentes résultent de la mise en parallèle incohérente d'un grand nombre de diodes Laser mais ne procurent pas la mise en phase du rayonnement émis par chacun de ces émetteurs élémentaires qui fournirait la cohérence spatiale d'un Laser unique et permettrait l'obtention d'un faisceau limité par la diffraction. On peut citer plusieurs approches à l'étude actuellement pour obtenir à la fois puissance et cohérence :

- Insertion des réseaux dans une cavité externe comportant un filtre spatial adéquat assurant le recouplage du rayonnement de chaque ruban élémentaire dans les autres et permettant leur synchronisation.

- Conception de réseaux serrés monolithiques où le couplage réparti entre les rubans élémentaires assure leur mise en phase [21].

- Synchronisation par verrouillage en fréquence des éléments d'un réseau de diodes Laser sur une diode Laser pilote.

- Intégration monolithique d'une diode Laser oscillateur maître et d'une diode Laser large fonctionnant en amplificateur de puissance, structure "MOPA".

\subsubsection{Etat de l'art en 1994}

Les chiffres les plus élevés en matière de puissance concernent des assemblages monolithiques ou hybrides de diodes Laser réalisées sur des substrats en GaAs, émettant par suite autour de $800 \mathrm{~nm}$ en régime incohérent et destinés au pompage de Lasers à Néodyme. On peut donner pour ces composants les chiffres repères suivants :

- 20 à $100 \mathrm{~W}$ en régime $\mathrm{CW}$ pour des barrettes linéaires monolithiques de longueur $1 \mathrm{~cm}$,

- 1 à $2 \mathrm{~kW}$ en régime QCW pour des assemblages surfaciques monolithiques ou hybrides de surface émissive voisine de $1 \mathrm{~cm}^{2}[23]$.

Les émetteurs monolithiques spatialement cohérents sont aujourd'hui limités vers $1 \mathrm{~W}$, mais les très nombreuses voies actuellement suivies pour repousser cette limite devraient permettre à moyen terme de tirer tout le bénéfice de Lasers à rendement de conversion d'énergie très élevé.

\subsection{Diodes Laser à rétroaction répartie [7]}

Une diode Laser PEROT-FABRY peut être considérée comme à rétroaction localisée, les faces clivées étant des miroirs situés aux extrémités de la cavité optique. Dans une diode Laser à rétroaction répartie de type DFB (Distributed FeedBack) ou de type DBR (Distributed BRAGG Reflector) la fonction de rétroaction est assurée par un réseau périodique gravé directement sur la région active dans le cas DFB illustré par la figure 27 ou sur un guide situé en bout de la région active dans le cas DBR.

Le rôle de ce réseau est de sélectionner un seul mode longitudinal de sorte que le spectre d'émission d'une telle diode Laser est dit monofréquence. Ce résultat est obtenu lorsque le pas du réseau $\Lambda$ satisfait la condition suivante :

$$
2 \Lambda \mathrm{n} / \lambda_{\mathrm{k}}=\mathrm{k}
$$

avec les mêmes notations qu'au $\S 6.4$, le nombre entier $k$ valant alors en pratique 1 (réseau du premier ordre) ou 2 (réseau du deuxième ordre).

Le résultat fonctionnel est une amélioration très importante de la cohérence temporelle, ou en d'autres termes, de la largeur spectrale du mode longitudinal unique. Elle est déterminée physiquement par l'émission spontanée ajoutée en quadrature à l'émission stimulée et faisant 
varier la phase de façon aléatoire. Son ordre de grandeur, exprimé en fréquence, est donné pour une diode Laser DFB typique par la relation :

\section{$\Delta \mathrm{f} \times \mathrm{P} \# 100 \mathrm{MHz} \times \mathrm{mW}$}

où $\Delta \mathrm{f}$ est la largeur spectrale en $\mathrm{MHz}$ et $\mathrm{P}$ la puissance émise en $\mathrm{mW}$.

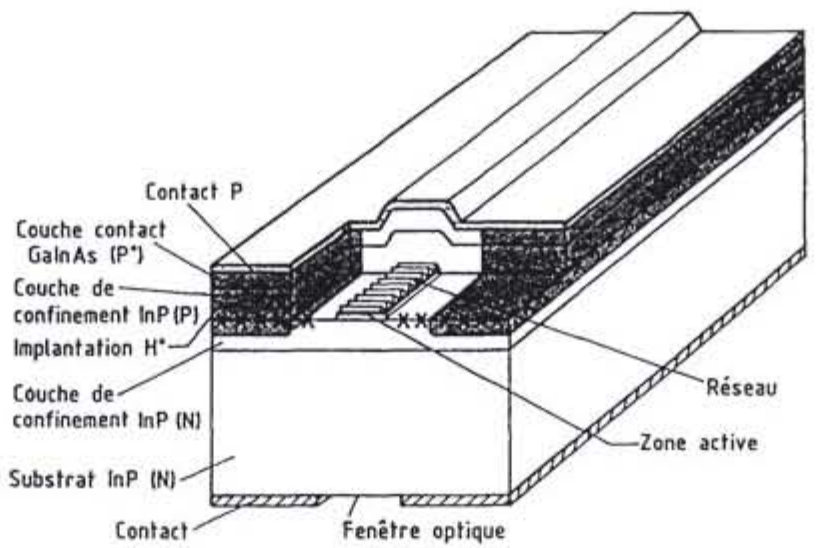

Figure 27 : Diode Laser à rétro-action répartie ou DFB (Distributed FeedBack).

Il est possible de réduire encore $\Delta \mathrm{f}$ par l'utilisation de cavités externes.

Le réglage en longueur d'onde du mode unique par une commande électrique et sur une plage plus large (quelques nm) que ce qui est accessible avec une commande en température (cf. § 6.4) peut être obtenu avec des dispositifs à 2 ou 3 sections réparties sur le même guide et commandées par des électrodes indépendantes, permettant d'ajuster séparément

- le gain d'une section amplificatrice,

- la longueur d'onde d'un réflecteur de BRAGG,

- et le déphasage entre les deux sections précédentes.

\subsection{Diodes superluminescentes}

Alors que les structures DFB et DBR visent à l'obtention d'une cohérence temporelle élevée, certaines applications demandent au contraire une cohérence temporelle faible tout en conservant une bonne cohérence spatiale afin de pouvoir coupler efficacement le rayonnement émis dans une fibre optique monomode. Les diodes superluminescentes (ou superradiantes), dont un mode de réalisation est schématisé sur la figure 28, permettent d'obtenir ce résultat.

La figure montre que la rétroaction des facettes est quasi annulée par l'utilisation d'un traitement diélectrique antireflet sur la facette "avant" et d'une région non excitée, et par suite absorbante, entre la région active et la facette "arrière". Le dispositif fonctionne alors comme un amplificateur d'émission spontanée avec un spectre d'émission semblable à celui d'une diode électroluminescente et un diagramme de directivité semblable à celui d'une diode Laser.

Il est ainsi possible d'atteindre à $850 \mathrm{~nm}$ une largeur spectrale de $30 \mathrm{~nm}$ et de coupler plus de $1 \mathrm{~mW}$ dans une fibre optique monomode. 


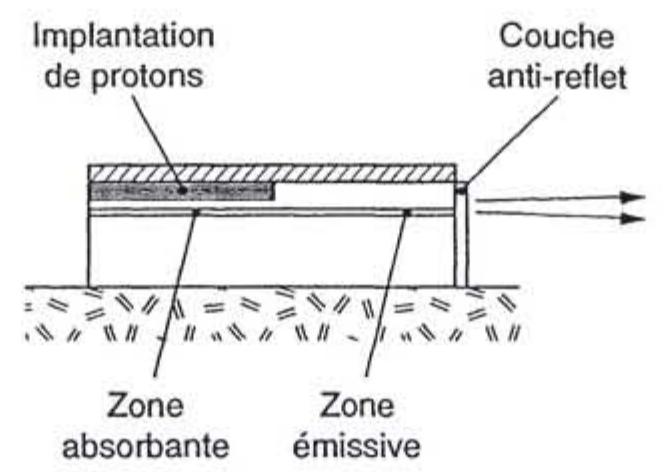

Figure 28 : Diode superluminescente.

\subsection{Extension du domaine de longueurs d'onde}

On a vu que la longueur d'onde d'émission d'un Laser à semi-conducteur dépend essentiellement du matériau constituant sa région active. La réalisation d'une diode Laser, dont le courant de seuil autorise un fonctionnement à température ambiante, suppose que soit élaborée de plus une structure à hétérojonctions avec des matériaux dont les gaps et indices de réfraction permettent d'assurer les confinements électronique et optique, et convenablement dopés pour injecter les électrons et les trous.

Les premières familles matériau/substrat ayant satisfait à ces conditions ont été GaAlAs/GaAs pour la fenêtre $750-900 \mathrm{~nm}$, puis GaInAsP/InP pour la "deuxième" fenêtre des télécommunications optiques $1100-1600 \mathrm{~nm}$. La bande $900-1100 \mathrm{~nm}$ située entre ces deux fenêtres a été ensuite comblée par l'extension de la famille GaAlAs/GaAs au moyen de puits quantiques contraints en GaInAs.

L'obtention des longueurs d'onde visibles a d'abord été recherchée par l'utilisation de matériaux épitaxiés avec accord de maille sur GaAs. Le matériau actif est GaInP et les couches de confinement sont les quaternaires AlGaInP, il est ainsi possible d'accéder aux longueurs d'ondes du rouge jusqu'à $630 \mathrm{~nm}$ [24]. La technologie de cette famille de matériaux atteint une maturité industrielle attestée par des niveaux de puissance $(60 \mathrm{~W},[25])$ et de fiabilité $\left(10^{6} \mathrm{H}\right.$ à $\left.50^{\circ} \mathrm{C},[26]\right)$ approchant ceux obtenus dans le proche infrarouge.

Les diodes Laser à longueur d'onde encore plus courtes sont encore des composants en étude. Les composés II-VI de la famille $\mathrm{CdMgZnSSe}$ épitaxiés sur GaAs semblent être les plus prometteurs pour l'émission dans le bleu-vert, et le fonctionnement continu à température ambiante et à $490 \mathrm{~nm}$ a été obtenu pour la première fois en 1993 [27]. Cependant les durées de vie des composants de cette famille sont encore très faibles : quelques dizaines de $\mathrm{s}$ à $1 \mathrm{~mW}$ [28]. Les II-V de la famille AlGaInN élaborés par épitaxie sur $\mathrm{Al}_{2} \mathrm{O}_{3}$ sont considérés pour l'émission jusqu'au proche UV mais n'ont démontré du gain d'émission stimulée qu'en pompage optique sous des densités de puissance très élevées : $200 \mathrm{~kW} / \mathrm{cm}^{2}$ pour $370 \mathrm{~nm}$ [29].

Les matériaux de la famille GaInAsP/InP permettent, par l'utilisation de puits quantiques contraints en GaInAs, d'étendre leur domaine spectral jusqu'à $2000 \mathrm{~nm}$ sans dégradation significative de leurs performances [30]. Au delà, il est nécessaire de faire appel aux 
hétérostructures de type GaInAsSb/AlGaAsSb/GaSb qui permettent l'obtention du fonctionnement continu à $2100 \mathrm{~nm}$ à $300 \mathrm{~K}$ [31] et à 3000 et $4000 \mathrm{~nm}$ respectivement à 170 et $80 \mathrm{~K}[32,33]$.

Les longueurs d'ondes de l'infrarouge moyen sont atteintes essentiellement avec les matériaux IV-VI du type $\mathrm{PbSnSeTe}$. Elles sont disponibles pour la spectroscopie depuis plusieurs années [34] et continuent à faire l'objet de développements technologiques [35].

\section{CONCLUSION}

Les diodes Laser présentent par rapport aux autres LASERS plusieurs différences fondamentales que l'on résume en conclusion ci-après.

- La densité d'atomes actifs est celle de la matière condensée, voisine de $10^{22} \mathrm{~cm}^{-3}$, ce qui permet d'obtenir des gains d'émission stimulée très élevés et par suite des dispositifs très compacts.

- Le pompage par injection dans une structure de diode est un procédé dont le rendement énergétique est très élevé puisque le courant d'alimentation est fourni sous une tension voisine de $\mathrm{hv} / \mathrm{q}$ et que le rendement quantique interne peut approcher $100 \%$ : l'énergie à fournir pour injecter un électron est voisine de celle du photon émis. De plus la modulation par injection directe permet d'obtenir simplement des signaux optiques modulés en amplitude à grande vitesse.

- Leur technologie de fabrication collective est celle de composants à semi-conducteurs et par suite leur coût unitaire décroît très vite avec les quantités produites. Compte tenu des nombreuses applications potentielles il apparait clairement que ces coûts vont encore décroître de façon significative, la vitesse de décroissance étant limitée par l'importance des moyens industriels à mettre en place. Cette évolution devrait être particulièrement manifeste à moyen terme pour les diodes de pompage de Laser solides.

Par ailleurs la diode Laser reste un sujet de recherches très actif. Les travaux en cours continuent d'étendre le domaine des performances accessibles, puissance, longueur d'onde, cohérence etc. Les difficultés majeures surviennent lorsque l'on cherche à réunir plusieurs caractéristiques extrêmes comme puissance et cohérence spatiale, longueur d'onde "courte" $e t$ fiabilité, ou encore longueur d'onde "grande" et température ambiante de fonctionnement. Les progrès actuels sont néanmoins très rapides et la réunion de caractéristiques qui apparaissent aujourd'hui contradictoires sera sans doute possible demain.

\section{Références}

[1] Dupuis R.D, IEEE J. Quantum Electron., QE-23 (June 1987), 651-657.

[2] Redike R.H., IEEE J. Quantum Electron., QE-23 (June 1987), 692-695.

[3] Anderson S.G., Laser Focus World, 30 (January 1994), 62-76.

[4] Sze S.M., Physics of Semiconductor Devices, 2nd Edition (John Wiley \& Sons, 1981)

[5] Mathieu H., Physique des semiconducteurs et des composants électroniques (Masson, 1987)

[6] Casey H.C., jr. and Panish M.B., Heterostructure Lasers, Part A - Fundamental principles, Part B - Materials and operating characteristics (Academic Press, 1978).

[7] Agrawall G.P. and Dutta N.K., Long-wavelength semiconductor Lasers (Van Nostrand, 1986).

[8] Peterman K., Laser diode modulation and noise (Kluwer Academic Publishers, 1988).

[9] Lau K.Y., IEEE J. Quantum Electron., QE-26 (1990), 250-261 
[10] Deryagin A.G. et al., Electron. Lett., 30 (1994), 309-311

[11] Thomson G.H.B. and Kirkby P.A., IEEE J. Quantum Electron., QE-9 (1990), 311

[12] Weisbuch C. and Vinter B., Quantum Semiconductor Stuctures: Fundamentals and Applications (Academic Press, Boston, 1991)

[13] Geels R.S., Thibeault B.J., Corzine S.W., Scott J.W. and Coldren L.A., IEEE J. Quantum Electron., 29 (1993), 2977-2987

[14] Young D.B., Kapila A., Scott J.W., Malhotra V. and Coldren L.A., Electron. Lett., 30 (1994), 233-235

[15] Orenstein M., Kapon E., Harbison J.P., Florez L.T. and Stoffel N.G., Appl. Phys. Lett., 60 (1992), 1535-1537

[16] Dudley J.J. and Bowers J.E., Appl. Phys. Lett., 64 (1994), 1463-1465

[17] Lott J.A., Schneider R.P., Choquette K.D., Kilcoyne S.P. and Figiel J.J., Electron. Lett., 29 (1993), 1693-1694

[18] Yamamoto Y. and Slusher R.E., Physics Today (June 1993), 66-73 